\title{
BOUNDEDNESS OF COMPLETELY ADDITIVE MEASURES WITH APPLICATION TO 2-LOCAL TRIPLE DERIVATIONS
}

\author{
JAN HAMHALTER, KARIMBERGEN KUDAYBERGENOV, ANTONIO M. PERALTA, \\ AND BERNARD RUSSO
}

\begin{abstract}
We prove a Jordan version of Dorofeev's boundedness theorem for completely additive measures and use it to show that every (not necessarily linear nor continuous) 2-local triple derivation on a continuous $\mathrm{JBW}^{*}$-triple is a triple derivation.
\end{abstract}

\section{INTRODUCTION AND BACKGROUND}

Let $\mathcal{P}(M)$ denote the lattice of projections in a von Neumann algebra $M$. Let $X$ be a Banach space. A mapping $\mu: \mathcal{P}(M) \rightarrow X$ is said to be finitely additive when

$$
\mu\left(\sum_{i=1}^{n} p_{i}\right)=\sum_{i=1}^{n} \mu\left(p_{i}\right),
$$

for every family $p_{1}, \ldots, p_{n}$ of mutually orthogonal projections in $M$. A mapping $\mu: \mathcal{P}(M) \rightarrow$ $X$ is said to be bounded when the set

$$
\{\|\mu(p)\|: p \in \mathcal{P}(M)\}
$$

is bounded.

The celebrated Bunce-Wright-Mackey-Gleason theorem ( [10], [11]) states that if $M$ has no summand of type $I_{2}$, then every bounded finitely additive mapping $\mu: \mathcal{P}(M) \rightarrow X$ extends to a bounded linear operator from $M$ to $X$.

Answering a question posed by George Mackey, Gleason's original theorem [21] characterizes quantum mechanical states on a separable Hilbert space in terms of density operators, and thus plays an important role in the foundations of quantum mechanics. The interdisciplinary nature of the Bunce-Wright-Mackey-Gleason theorem makes this result very useful in a wide range of topics. Applications can be found in quantum physics and quantum information (cf. [18], [43], [37], [36], [22, Chapter 7], and [16], among many others), and in functional analysis with studies on vector-valued measures on von Neumann

1991 Mathematics Subject Classification. Primary 46L70; 46L57; 46L40.

Key words and phrases. triple derivation; 2-local triple derivation, Gleason theorem, continuous JBW*triple.

First named author was supported by the "Grant Agency of the Czech Republic" grant number P201/12/0290, "Topological and geometrical properties of Banach spaces and operator algebras". Third named author was partially supported by the Spanish Ministry of Economy and Competitiveness project no. MTM2014-58984-P and Junta de Andalucía grant FQM375. 
algebras and 2-local maps on von Neumann algebras, $\mathrm{JBW}^{*}$-algebras and $\mathrm{JBW}^{*}$-triples (see [20], [3], [4], [13] [14] and [32]).

According to the terminology employed in [39] and [17], a completely additive mapping $\mu: \mathcal{P}(M) \rightarrow \mathbb{C}$ - that is, (1.1) holds with $X=\mathbb{C}$ for an arbitrary set of mutually orthogonal projections, is called a charge. The Dorofeev-Sherstnev theorem ( [39, Theorem 29.5] or [17, Theorem 2]) states that any charge on a von Neumann algebra with no summands of type $I_{n}$ is bounded.

The Dorofeev-Shertsnev theorem was used in [32] in order to apply the Bunce-WrightMackey-Gleason theorem to prove the main result of that paper, namely, that a 2-local triple derivation on a von Neumann algebra is a triple derivation (see the next subsection). In section 3 of this paper, we shall establish the first main result of this paper, namely, a Jordan version of Dorofeev's boundedness theorem (Theorem 3.1). This will be used in section 4 to show that 2-local triple derivations on certain continuous $\mathrm{JW}^{*}$-algebras are triple derivations (Theorem 4.6). Combined with the main result of section 2 (Theorem 2.4), this will prove the second main result of this paper, namely, that every 2-local triple derivation on an arbitrary continuous $\mathrm{JBW}^{*}$-triple is a triple derivation (Theorem 4.7).

Having described the contents and potential impact of this paper, we shall now present more background and some preliminary material.

We shall use the term measure to denote a complex valued finitely additive function $\mu$ on the projections of a von Neumann algebra or a $\mathrm{JBW}^{*}$-algebra. If $\mu$ is positive (resp. real) valued, we call it a positive (resp. signed) measure. If countable additivity or complete additivity is assumed, it will be explicitly stated.

Let us recall that a derivation is a linear map $D$ from an algebra $A$ to a two sided $A$ module $M$ over the algebra satisfying the Leibniz identity: $D(a b)=a \cdot D(b)+D(a) \cdot b$ for all $a, b \in A$.

Local derivations were introduced simultaneously in 1990 by Kadison [28] and by LarsonSourour [33]. A local derivation from an algebra into a module is a linear mapping whose value at each point in the algebra coincides with the value of some derivation at that point. Kadison proved that every continuous local derivation of a von Neumann algebra into a dual Banach module is in fact a derivation. Johnson [27] extended Kadison's result to $\mathrm{C}^{*}$-algebras, and moreover showed that the continuity assumption was not necessary. Larson and Sourour showed that a local derivation on the algebra of all bounded linear operators on a Banach space is a derivation.

Let us also recall that a triple derivation is a linear map $D$ from a triple system $E$ to an $E$-module $N$ over the triple system satisfying the triple Leibniz identity: $D(\{a b c\})=$ $\{D(a) b c\}+\{a D(b) c\}+\{a b D(c)\}$ for all $a, b, c \in E$, where $\{a b c\}$ denotes the triple product. (Jordan triple systems are defined later in this section.)

Local triple derivations were introduced in 2013 by Michael Mackey [34]. A local triple derivation on a triple system is a linear mapping whose value at each point in the triple system coincides with the value of some triple derivation at that point. Mackey showed that a continuous local triple derivation on a $\mathrm{JBW}^{*}$-triple (to itself) is a triple derivation, an 
exact analog of Kadison's result mentioned above. This result was extended to JB*-triples in 2014 by Burgos, Fernandez-Polo, and Peralta [12], who also showed that the continuity assumption was redundant, an exact analog of Johnson's result also mentioned above.

Since 1997 there has been much interest in the notion of 2-local derivation and more recently, in the notion of 2-local triple derivation. The application of the main theorem of this paper concerns 2-local triple derivations. A 2-local derivation (respectively, 2-local triple derivation) from an algebra (respectively, triple system) into itself is a mapping (not necessarily linear) whose values at each pair of points in the algebra (respectively, triple system) coincides with the values of some derivation (respectively, triple derivation) at those two points. 2-local derivations were introduced in 1997 by Semrl [38] and 2-local triple derivations were introduced in 2014 by Kudaybergenov, Oikhberg, Peralta, and Russo [32] although the concept was mentioned by Michael Mackey in a lecture in 2012 at a conference in Hong Kong celebrating the 65th birthday of Cho-Ho Chu. It is now known that, for von Neumann algebras, a 2-local derivation is in fact a derivation (Ayupov-Kudaybergenov [3]) and, as noted above, a 2-local triple derivation is a triple derivation (KudaybergenovOikhberg-Peralta-Russo [32]).

For an elaboration of the above summary, see the forthcoming survey of Ayupov, $\mathrm{Ku}$ daybergenov, and Peralta, [4]. Local and 2-local derivations have also been considered on algebras of measurable operators associated with von Neumann algebras. For more details on this, see the forthcoming survey of Ayupov and Kudaybergenov [2].

A complex Jordan triple is a complex vector space $E$ equipped with a non-trivial triple product

$$
\begin{gathered}
E \times E \times E \rightarrow E \\
(x, y, z) \mapsto\{x, y, z\}
\end{gathered}
$$

which is bilinear and symmetric in the outer variables and conjugate linear in the middle one satisfying the so-called "Jordan Identity":

$$
L(a, b) L(x, y)-L(x, y) L(a, b)=L(L(a, b) x, y)-L(x, L(b, a) y),
$$

for all $a, b, x, y$ in $E$, where $L(x, y) z:=\{x, y, z\}$.

A subspace $F$ of a Jordan triple $E$ is said to be a subtriple if $\{F, F, F\} \subseteq F$ and an ideal if $\{E, E, J\}+\{E, J, E\} \subseteq J$.

A (complex) $J B^{*}$-triple is a complex Jordan Banach triple $E$ satisfying the following axioms:

- For each $a$ in $E$ the map $L(a, a)$ is an hermitian operator on $E$ with non negative spectrum;

- $\|\{a, a, a\}\|=\|a\|^{3}$ for all $a$ in $A$.

A JB*-algebra is a complex Jordan Banach algebra $(A, \circ)$ equipped with an algebra involution * satisfying $\left\|\left\{a, a^{*}, a\right\}\right\|=\|a\|^{3}, a \in A$. (Recall that $\left\{a, a^{*}, a\right\}=2\left(a \circ a^{*}\right) \circ a-a^{2} \circ a^{*}$.) JB-algebras are precisely the self adjoint parts of $\mathrm{JB}^{*}$-algebras, and a JBW-algebra is a JBalgebra which is a dual space. 
Every $\mathrm{C}^{*}$-algebra (resp., every $\mathrm{JB}^{*}$-algebra) is a $\mathrm{JB}^{*}$-triple with respect to the product $\{a, b, c\}=\frac{1}{2}\left(a b^{*} c+c b^{*} a\right)$ (resp., $\left.\{a, b, c\}:=\left(a \circ b^{*}\right) \circ c+\left(c \circ b^{*}\right) \circ a-(a \circ c) \circ b^{*}\right)$.

For the theory of $\mathrm{C}^{*}$-algebras and von Neumann algebras, we shall refer to the monographs [29] and [40]. For the theory of $\mathrm{JB}^{*}$-algebras and $\mathrm{JBW}^{*}$-algebras we refer to [23] and [41]. For basic facts about abstract Jordan triple systems, consult [15, section 1.2]. However, the Jordan triple systems we consider in this paper are concrete, so statements about them can usually be verified directly. For example, a tripotent (defined in the next section) is nothing but a partial isometry.

A complex $J B W^{*}$-triple is a complex $\mathrm{JB}^{*}$-triple which is also a dual Banach space. The structure of $\mathrm{JBW}^{*}$-triples is fairly well understood. Every $\mathrm{JBW}^{*}$-triple is a direct sum of a $\mathrm{JBW}^{*}$-triple of type I and a continuous $\mathrm{JBW}^{*}$-triple (defined below). $\mathrm{JBW}^{*}$-triples of type I have been classified in [25] and continuous $\mathrm{JBW}^{*}$-triples have been classified in [26]. Since it is the continuous $\mathrm{JBW}^{*}$-triples that concern us here, we shall not define type I, but we shall state their classification theorem from [25]: A $\mathrm{JBW}^{*}$-triple of type I is an $\ell^{\infty}$-direct sum of $\mathrm{JBW}^{*}$-triples of the form $A \otimes C$, where $A$ is a commutative von Neumann algebra and $C$ is a Cartan factor (for Cartan factors, see [15, Theorem 2.5.9 and page 168]).

A $J B W^{*}$-triple $\mathcal{A}$ is said to be continuous if it has no type I direct summand. In this case it is known that, up to isometry, $\mathcal{A}$ is a $J W^{*}$-triple, that is, a subspace of the bounded operators on a Hilbert space which is closed under the triple product $x y^{*} z+z y^{*} x$ and closed in the weak operator topology. More importantly, it has a unique decomposition into weak ${ }^{*}$-closed ideals, $\mathcal{A}=H(W, \alpha) \oplus p V$, where $W$ and $V$ are continuous von Neumann algebras, $p$ is a projection in $V, \alpha$ is an involution on $W$ commuting with ${ }^{*}$, that is, a *antiautomorphism of $W$ order 2, which we shall call henceforth a $\mathbb{C}$-linear *-involution, and $H(W, \alpha)=\{x \in W$ : $\alpha(x)=x\}$ (see [26, (1.20)]). Notice that the triple product in $p V$ is given by $\left(x y^{*} z+z y^{*} x\right) / 2$ and that $H(W, \alpha)$ is a $\mathrm{JBW}^{*}$-algebra with the Jordan product $x \circ y=(x y+y x) / 2$.

We shall show in section 4 that for continuous $\mathrm{JBW}^{*}$-triples, every 2-local triple derivation is a derivation. (We are leaving the study of 2-local triple derivations on the $\mathrm{JBW}^{*}$-triples of type I as one of the problems at the end of this paper-see Problem 4.9(a).)

\section{2-LOCAL TRIPLE DERIVATIONS ON RIGHT IDEALS OF VON NEUMANN ALGEBRAS}

Recall that a (not necessarily linear) mapping $\Delta$ on a Jordan triple $E$ is said to be a 2-local triple derivation if, given two points $x, y \in E$, there is a triple derivation $D_{x, y}$ on $E$ such that $\Delta(x)=D_{x, y}(x)$ and $\Delta(y)=D_{x, y}(y)$. Every 2-local triple derivation $\Delta: E \rightarrow E$ is homogeneous. Indeed, for each $a \in E, t \in \mathbb{C}$ consider a triple derivation $D_{a, t a}$. Then $\Delta(t a)=D_{a, t a}(t a)=t D_{a, t a}(a)=t \Delta(a)$.

An element $e$ in a Jordan triple $E$ is called a tripotent if $\{e, e, e\}=e$. Each tripotent $e$ in $E$ induces a decomposition of $E$ (called Peirce decomposition) in the form:

$$
E=E_{0}(e) \oplus E_{1}(e) \oplus E_{2}(e),
$$

where $E_{k}(e)=\left\{x \in E: L(e, e) x=\frac{k}{2} x\right\}$ for $k=0,1,2$ (compare [15, page 32]). 
Lemma 2.1. Let $\Delta: \mathcal{A} \rightarrow \mathcal{A}$ be a 2-local triple derivation on a JB*-triple. Suppose $v$ is a tripotent in $\mathcal{A}$ such that $\Delta(v)=0$. Then $\Delta\left(\mathcal{A}_{k}(v)\right) \subseteq \mathcal{A}_{k}(v)$, for every $k=0,1,2$.

Proof. Let $x \in \mathcal{A}_{k}(v)$ with $k=0,1,2$, that is, $\{v, v, x\}=\frac{k}{2} x$. Since

$$
\begin{aligned}
\{v, v, \Delta(x)\} & =\left\{v, v, D_{v, x}(x)\right\}=D_{v, x}(\{v, v, x\})-\left\{D_{v, x}(v), v, x\right\}-\left\{v, D_{v, x}(v), x\right\}= \\
& =D_{v, x}\left(\frac{k}{2} x\right)-\{\Delta(v), v, x\}-\{v, \Delta(v), x\}=\frac{k}{2} D_{v, x}(x)=\frac{k}{2} \Delta(x) .
\end{aligned}
$$

The proof is complete.

We recall the following result (see [32, Theorem 2.14]).

Theorem 2.2. [32] Let $M$ be an arbitrary von Neumann algebra and let $T: M \rightarrow M$ be a (not necessarily linear nor continuous) 2-local triple derivation. Then $T$ is a triple derivation.

Throughout this section $\mathcal{A}$ will denote the $J B W^{*}$-triple $p M$ where $M$ is a von Neumann algebra and $p$ is a projection in $M$. The following is the main result of this section. The proof will be carried out in the next subsections.

Theorem 2.3. Let $M$ be a von Neumann algebra and let $p$ be a projection in $M$. Then any 2-local triple derivation $\Delta$ on the $J B W^{*}$-triple $\mathcal{A}=p M$ is a triple derivation.

Let $a$ and $b$ be skew-hermitian elements in $p M p$ and $M$, respectively. Let $L_{a}$ and $R_{b}$ be the left and right multiplication operators, i.e.

$$
L_{a}(x)=a x, x \in \mathcal{A}
$$

and

$$
R_{b}(x)=x b, x \in \mathcal{A} .
$$

It is clear that $L_{a}$ and $R_{b}$ both are triple derivations on $M$, and in particular on $\mathcal{A}$.

Let $u$ be a tripotent in the $J B W^{*}$-triple $\mathcal{A}=p M$, and let $\left(\mathcal{A}_{2}(u),{ }^{\cdot} u,{ }^{{ }^{*} u}\right)$ denote the von Neumann algebra whose underlying Banach space is the Pierce-2-space $\mathcal{A}_{2}(u)=u u^{*} M u^{*} u$, and whose product and involution are given by $x \cdot{ }_{u} y=x u^{*} y$ and $x^{* u}=u x^{*} u$, respectively.

Let $\{., ., .\}_{1}$ denote the triple product associated to $\mathcal{A}_{2}(u)$, i.e. $\{x, y, z\}_{1}=\frac{1}{2}\left(x \cdot{ }_{u} y^{* u} \cdot{ }_{u}\right.$ $\left.z+z \cdot{ }_{u} y^{*} u \cdot{ }_{u} x\right)$. By direct calculation, $\{x, y, z\}_{1}=\{x, y, z\}$. This also follows since the identity map is a linear isometry, and therefore an isomorphism ( [31, Proposition (5.5)]). Therefore a linear mapping $D: \mathcal{A}_{2}(u) \rightarrow \mathcal{A}_{2}(u)$ is a triple derivation (resp. 2-local triple derivation) for the product $\{., .,$.$\} if and only if it is a triple derivation (resp. 2-local triple$ derivation) for the product $\{., ., .\}_{1}$.

2.1. Properly infinite case. In this subsection we will consider 2-local triple derivations on $J B W^{*}$-triples of the form $\mathcal{A}=p M$, where $p$ is a properly infinite projection in a von Neumann algebra $M$. 
Let $q$ be a projection in $M$ and let $D$ be a triple derivation on $\mathcal{A}=p M$. It is easily seen that an operator $D_{(q)}$ on the $J B W^{*}$-subtriple $p M q$ defined by

$$
D_{(q)}(x)=D(x) q, x \in p M q
$$

is a triple derivation on $p M q$. Thus, if $\Delta$ is a 2-local triple derivation on $\mathcal{A}=p M$, then the operator $\Delta_{(q)}$ on the $J B W^{*}$-subtriple $p M q$ defined by

$$
\Delta_{(q)}(x)=\Delta(x) q, x \in p M q
$$

is a 2-local triple derivation on $p M q$.

The following is the main result of this subsection.

Theorem 2.4. Let $M$ be a von Neumann algebra and let $p$ be a properly infinite projection in $M$. Then any 2-local triple derivation $\Delta$ on $\mathcal{A}=p M$ is a triple derivation.

Proof. Since $p$ is properly infinite, by using the halving Lemma five times (see for example [29, Lemma 6.3.3]) we can find mutually orthogonal projections $e_{1}, \ldots, e_{6}$ in $M$ such that $p \sim e_{1} \sim \ldots \sim e_{6}$ and $p=e_{1}+\cdots+e_{6}$.

Denote by $r(x)$ and $l(x)$ the right and left supports in $M$ of the element $x$ from $M$, respectively. Since $r(x) \sim l(x)$ (see [40, Proposition V.1.5]) and $l(x) \leq p$, it follows that $r(x) \preceq p$ for all $x \in \mathcal{A}$.

Let $x, y \in \mathcal{A}$. Denote by $q_{1}, \ldots, q_{6}$ the right supports of elements $x, y, x+y, \Delta(x)$, $\Delta(y)$ and $\Delta(x+y)$, respectively. Then $q_{i} \preceq p$ for all $i \in\{1, \ldots, 6\}$. Since $p \sim e_{i}$ for all $i$, it follows that $q_{i} \preceq e_{i}$ for all $i \in\{1, \ldots, 6\}$. Therefore $\bigvee_{i=1}^{6} q_{i} \preceq e_{1}+\ldots+e_{6}=p$ (see [30, Exercise 6.9.3]).

Let us show the existence of a projection $q \in M$ such that $\bigvee_{i=1}^{6} q_{i} \leq q \sim p=e_{1}+\cdots+e_{6}$. Since $p$ is properly infinite by [30, Exercise 6.9.4] it follows that

$$
\left(\bigvee_{i=1}^{6} q_{i}\right) \vee p \sim p
$$

Then it suffices to take $q=\left(\bigvee_{i=1}^{6} q_{i}\right) \vee p$.

Since $p \sim q$ there exists a partially isometry $u \in M$ such that $u u^{*}=p, u^{*} u=q$. As was mentioned before this subsection, $p M q=u u^{*} M u^{*} u$ is a von Neumann algebra with respect to product and involution given by $x \cdot{ }_{u} y=x u^{*} y$ and $x^{*} u=u x^{*} u$, respectively.

Let $\Delta_{(q)}$ be the 2-local triple derivation on $p M q$ defined by (2.3). Then by Theorem 2.2, $\Delta_{(q)}$ is a triple derivation. By the construction of $q$ it follows that $x, y, x+y, \Delta(x), \Delta(y), \Delta(x+$ $y$ ) all belong to $p M q$. Therefore

$$
\begin{aligned}
\Delta(x+y) & =\Delta(x+y) q=\Delta_{(q)}(x+y)=\Delta_{(q)}(x)+\Delta_{(q)}(y)= \\
& =\Delta(x) q+\Delta(y) q=\Delta(x)+\Delta(y) .
\end{aligned}
$$


Thus $\Delta$ is additive and hence linear. Since every (linear) local triple derivation on a JB*triple is automatically continuous and hence a triple derivation (see [12, Theorem 2.8]), the proof is complete.

2.2. Finite case. In this subsection we will consider 2-local triple derivations on $J B W^{*}$ triples of the form $\mathcal{A}=p M$, where $p$ is a finite projection in a von Neumann algebra $M$.

Let $D$ be a triple derivation on $\mathcal{A}$. Set, for a tripotent $u \in \mathcal{A}$,

$$
D^{(u)}(x)=\{u,\{u, D(x), u\}, u\}=u u^{*} D(x) u^{*} u, x \in \mathcal{A}_{2}(u) .
$$

It is easily seen that $D^{(u)}$ is a triple derivation on $\mathcal{A}_{2}(u)$.

Let $\Delta$ be a 2-local triple derivation on $\mathcal{A}$ and let $u$ be a tripotent in $\mathcal{A}$. Then

$$
u u^{*} \Delta(u) u^{*} u=-u \Delta(u)^{*} u \text {. }
$$

Indeed, take a triple derivation $D$ on $\mathcal{A}$ with $\Delta(u)=D(u)$. From the equality $\{u, u, u\}=$ $u$, we have that

$$
u u^{*} D(u) u^{*} u=-u D(u)^{*} u
$$

which implies (2.5).

Lemma 2.5. Let $\Delta$ be a 2-local derivation on $\mathcal{A}$. There exist skew-hermitian elements $a_{1}$ in $p M p$ and $b_{1}$ in $M$ such that

$$
\Delta(p)=L_{a_{1}}(p)+R_{b_{1}}(p)
$$

Proof. Set

$$
a_{1}=\Delta(p) p \text { and } b_{1}=\Delta(p) p^{\perp}-p^{\perp} \Delta(p)^{*},
$$

where $p^{\perp}=\mathbf{1}-p$. From (2.5) it follows that $a_{1}$ is skew-hermitian. It is clear that $b_{1}$ is also skew-hermitian. We have

$$
L_{a_{1}}(p)+R_{b_{1}}(p)=a_{1} p+p b_{1}=\Delta(p) p+p \Delta(p) p^{\perp}=\Delta(p) p+\Delta(p) p^{\perp}=\Delta(p) .
$$

Lemma 2.6. Let $\Delta$ be a 2-local derivation on $\mathcal{A}$. Suppose that $\Delta(p)=0$. Then there exists a skew-hermitian element $a_{2}$ in $p M p$ such that $\Delta(x)=L_{a_{2}}(x)-R_{a_{2}}(x)$ for all $x \in \mathcal{A}_{2}(p)=$ $p M p$.

Proof. Since $\Delta(p)=0$, Lemma 2.1 implies that $\Delta$ maps $\mathcal{A}_{2}(p)=p M p$ into itself.

Let $x, y \in \mathcal{A}_{2}(p)$. Take a triple derivation $D_{x, y}$ on $\mathcal{A}$ such that

$$
\Delta(x)=D_{x, y}(x), \Delta(y)=D_{x, y}(y) .
$$

Let $D_{x, y}^{(p)}$ be the triple derivation defined by (2.4). Then

$$
\Delta(x)=D_{x, y}^{(p)}(x), \Delta(y)=D_{x, y}^{(p)}(y) .
$$

This means that the restriction $\left.\Delta\right|_{\mathcal{A}_{2}(p)}$ is a 2-local triple derivation on the von Neumann algebra $\mathcal{A}_{2}(p)$. By Theorem 2.2, $\left.\Delta\right|_{\mathcal{A}_{2}(p)}$, is a triple derivation. Since $\Delta(p)=0$, there exists a skew-hermitian element $a_{2}$ in $p M p$ such that $\Delta(x)=a_{2} x-x a_{2}$ for all $x \in \mathcal{A}_{2}(p)=p M p$ (see [32, beginning of section 2]). 
Let $D$ be an arbitrary triple derivation (or a 2-local triple derivation) on $\mathcal{A}$. Then $D$ can be decomposed in the form

$$
D=D_{1}+D_{2}
$$

where $D_{1}=L_{a}+R_{b}$, with $a, b$ skew-hermitian and $\left.D_{2}\right|_{\mathcal{A}_{2}(p)} \equiv 0$.

Indeed, by Lemma 2.5, there exist skew-hermitian elements $a_{1} \in p M p$ and $b_{1} \in M$ such that $\left(D-L_{a_{1}}-R_{b_{1}}\right)(p)=0$. By Lemma 2.6, there is a skew-hermitian element $a_{2} \in p M p$ such that $\left(D-L_{a_{1}}-R_{b_{1}}\right)(x)=L_{a_{2}} x-R_{a_{2}} x$ for all $x \in p M p$. Now it suffices to set

$$
D_{1}=L_{a_{1}+a_{2}}+R_{b_{1}-a_{2}} \text { and } D_{2}=D-D_{1} \text {. }
$$

Lemma 2.7. Let $D$ be a triple derivation on $\mathcal{A}$ such that $\left.D\right|_{\mathcal{A}_{2}(p)} \equiv 0$. Then

$$
D(x) y^{*}+x D(y)^{*}=0
$$

for all $x, y \in \mathcal{A}$.

Proof. Let us first consider a case $x, y \in \mathcal{A}_{1}(p)=p M(\mathbf{1}-p)$.

Since $\left.D\right|_{\mathcal{A}_{2}(p)} \equiv 0$, it follows from Lemma 2.1 that $D$ maps $\mathcal{A}$ into $\mathcal{A}_{1}(p)$. Taking into account these properties we have

$$
\begin{aligned}
x D(y)^{*} & =x D(y)^{*} p+p D(y)^{*} x=2\{x, D(y), p\}= \\
& =2 D(\{x, y, p\})-2\{D(x), y, p\}-2\{x, y, D(p)\}= \\
& =D\left(x y^{*} p+p y^{*} x\right)-D(x) y^{*} p-p y^{*} D(x)= \\
& =D\left(x y^{*}\right)-D(x) y^{*}=-D(x) y^{*},
\end{aligned}
$$

i.e. $D(x) y^{*}+x D(y)^{*}=0$ for $x, y \in \mathcal{A}_{1}(p)$.

Let now $x, y \in \mathcal{A}$ be arbitrary and let $x=x_{2}+x_{1}, y=y_{2}+y_{1} \in \mathcal{A}=\mathcal{A}_{2}(p)+\mathcal{A}_{1}(p)$. We have

$$
\begin{aligned}
D(x) y^{*}+x D(y)^{*} & =D\left(x_{2}+x_{1}\right)\left(y_{2}+y_{1}\right)^{*}+\left(x_{2}+x_{1}\right) D\left(y_{2}+y_{1}\right)^{*}= \\
& =D\left(x_{1}\right) y_{2}^{*}+x_{1} D\left(y_{1}\right)^{*}+D\left(x_{1}\right) y_{1}^{*}+x_{2} D\left(y_{1}\right)^{*} \\
& =D\left(x_{1}\right) y_{2}^{*}+x_{2} D\left(y_{1}\right)^{*}=0,
\end{aligned}
$$

because $D\left(x_{1}\right) y_{2}^{*}=\left(D\left(x_{1}\right)(\mathbf{1}-p)\right)\left(y_{2} p\right)^{*}=0$ and $x_{2} D\left(y_{1}\right)^{*}=x_{2} p\left(D\left(y_{1}\right)(\mathbf{1}-p)\right)^{*}=0$. The proof is complete.

Since $p M p$ is finite, there exists a faithful center-valued trace $\tau$ on $p M p$, that is, a linear map from $p M p$ into the center, $Z(p M p)$, of $p M p$ such that

(i) $\tau(x y)=\tau(y x)$ for all $x, y \in p M p$;

(ii) $\tau(z)=z$ for all $z \in Z(p M p)$;

(iii) $\tau\left(x x^{*}\right)=0$ implies $x=0$.

Define a $Z(p M p)$-valued sesquilinear form on $\mathcal{A}$ by

$$
\langle x, y\rangle=\tau\left(x y^{*}\right), x, y \in \mathcal{A} .
$$

Since $\tau$ is faithful it follows that the form $\langle\cdot, \cdot\rangle$ is non-degenerate, i.e. $\langle x, y\rangle=0$ for all $y \in \mathcal{A}$ implies that $x=0$. 
Lemma 2.8. Let $D$ be an arbitrary triple derivation on $\mathcal{A}$. Then

$$
\langle D(x), y\rangle=-\langle x, D(y)\rangle
$$

for all $x, y \in \mathcal{A}$.

Proof. Let $D=D_{1}+D_{2}$ be a decomposition of $D$ in the form (2.7). For $x, y \in \mathcal{A}$, we have $a^{*}=-a \in p M p$ and $b^{*}=-b \in M$ such that

$$
\begin{aligned}
D_{1}(x) y^{*}+x D_{1}(y)^{*} & =(a x+x b) y^{*}+x(a y+y b)^{*}= \\
& =a x y^{*}+x b y^{*}+x y^{*} a^{*}+x b^{*} y^{*}= \\
& =a x y^{*}+x b y^{*}-x y^{*} a-x b y^{*}= \\
& =a x y^{*}-x y^{*} a,
\end{aligned}
$$

i.e.

$$
D_{1}(x) y^{*}+x D_{1}(y)^{*}=a x y^{*}-x y^{*} a .
$$

Since a center-valued trace annihilates commutators we have that

$$
\tau\left(D_{1}(x) y^{*}+x D_{1}(y)^{*}\right)=0 .
$$

Thus

$$
\left\langle D_{1}(x), y\right\rangle=-\left\langle x, D_{1}(y)\right\rangle .
$$

On the other hand, by Lemma 2.7 it follows that

$$
\left\langle D_{2}(x), y\right\rangle=-\left\langle x, D_{2}(y)\right\rangle \text {. }
$$

The proof is complete.

The following is the main result of this subsection.

Theorem 2.9. Let $M$ be a von Neumann algebra and let $p$ be a finite projection in $M$. Then any 2-local triple derivation $\Delta$ on $\mathcal{A}=p M$ is a triple derivation.

Proof. Let us first show that

for all $x, y \in \mathcal{A}$.

$$
\langle\Delta(x), y\rangle=-\langle x, \Delta(y)\rangle
$$

Take a triple derivation $D$ on $\mathcal{A}$ such that

$$
\Delta(x)=D(x) \text { and } \Delta(y)=D(y) .
$$

By Lemma 2.8, we have

$$
\langle\Delta(x), y\rangle=\langle D(x), y\rangle=-\langle x, D(y)\rangle=-\langle x, \Delta(y)\rangle .
$$

Let now $x, y, z$ be arbitrary elements in $\mathcal{A}$. Then

$$
\begin{aligned}
\langle\Delta(x+y), z\rangle & =-\langle x+y, \Delta(z)\rangle=-\langle x, \Delta(z)\rangle-\langle y, \Delta(z)\rangle= \\
& =\langle\Delta(x), z\rangle+\langle\Delta(y), z\rangle=\langle\Delta(x)+\Delta(y), z\rangle,
\end{aligned}
$$

i.e.

$$
\langle\Delta(x+y)-\Delta(x)-\Delta(y), z\rangle=0 .
$$


Since $z$ is an arbitrary and the sesquilinear form is non-degenerate it follows that $\Delta(x+y)=$ $\Delta(x)+\Delta(y)$, so $\Delta$ is additive, hence linear, hence a triple derivation by [12, Theorem 2.8] (compare the proof of Theorem 2.4).

2.3. General case. We need the following two Lemmata.

Lemma 2.10. Let $D$ be a triple derivation on $p M$. Then $D$ is $\mathcal{P}(Z(M))$-homogeneous, i.e.

$$
D(c x)=c D(x)
$$

for any central projection $c \in \mathcal{P}(Z(M))$ and $x \in p M$.

Proof. Let $c \in \mathcal{P}(Z(M))$. Take $x, y, z \in p M$. We have

$$
\begin{aligned}
c\{x, D(c y), z\} & =c D(\{x, c y, z\})-c\{D(x), c y, z\}-c\{x, c y, D(z)\}= \\
& =c D(\{x, c y, z\})-c\{c D(x), y, z\}-c\{x, y, D(z)\}
\end{aligned}
$$

and

$$
\begin{aligned}
c\{x, D(c y), z\} & =c\{c x, D(c y), z\}= \\
& =c D(\{c x, c y, z\})-c\{D(c x), c y, z\}-c\{c x, c y, D(z)\}= \\
& =c D(\{x, c y, z\})-c\{D(c x), y, z\}-c\{x, y, D(z)\} .
\end{aligned}
$$

Thus $c\{c D(x), y, z\}=c\{D(c x), y, z\}$. Since $c$ is a central projection we obtain that

$$
\{c D(x), y, z\}=\{c D(c x), y, z\} .
$$

Since $y, z$ are arbitrary, it follows that

$$
c D(x)=c D(c x) .
$$

Thus

$$
c D((\mathbf{1}-c) x)=0 .
$$

Replacing $c$ by $\mathbf{1}-c$ in the last equality we obtain that

$$
(\mathbf{1}-c) D(c x)=0 \text {. }
$$

Thus

$$
D(c x)=(c+(\mathbf{1}-c)) D(c x)=c D(c x)+(\mathbf{1}-c) D(c x) \stackrel{(2.10)}{=} c D(c x) \stackrel{(2.9)}{=} c D(x) .
$$

The proof is complete.

Lemma 2.11. Let $\Delta$ be a 2-local triple derivation on $p M$. Then $\Delta$ is $\mathcal{P}(Z(M))$-homogeneous, i.e.

$$
\Delta(c x)=c \Delta(x)
$$

for any central projection $c \in \mathcal{P}(Z(M))$ and $x \in p M$.

Proof. Let $c \in \mathcal{P}(Z(M))$ and $x \in p M$. Let $D_{c x, x}: p M \rightarrow p M$ be a triple derivation satisfying $\Delta(c x)=D_{c x, x}(c x)$ and $\Delta(x)=D_{c x, x}(x)$. By Lemma 2.10, we have

$$
\Delta(c x)=D_{c x, x}(c x)=c D_{c x, x}(x)=c \Delta(x) .
$$


Now we are in position to prove Theorem 2.3.

Proof of Theorem 2.3. Let $M$ be a von Neumann algebra, $p$ be a projection in $M$ and $\Delta$ be a 2-local triple derivation on the $J B W^{*}$-triple $\mathcal{A}=p M$.

Take mutually orthogonal central projections $z_{1}$ and $z_{2}$ in $M$ with $z_{1}+z_{2}=1$ such that $z_{1} p$ is finite and $z_{2} p$ is properly infinite. Lemma 2.11 implies that $\Delta$ maps each $z_{i} \mathcal{A}$ into itself and hence induces a 2-local triple derivation $\Delta_{i}=\left.\Delta\right|_{z_{i} \mathcal{A}}$ on $z_{i} \mathcal{A}=z_{i} p M$ for $i=1,2$. Theorems 2.4, 2.9 imply that both $\Delta_{1}$ and $\Delta_{2}$ are triple derivations. Since

$$
\Delta(x)=z_{1} \Delta(x)+z_{2} \Delta(x)=\Delta_{1}\left(z_{1} x\right)+\Delta_{2}\left(z_{2} x\right)
$$

for all $x \in \mathcal{A}$, it follows that $\Delta$ is also a triple derivation. The proof is complete.

A Cartan factor of type 1 is the $\mathrm{JBW}^{*}$-triple $B(H, K)$ of all bounded operators from a Hilbert space $H$ to a Hilbert space $K$. We thus have:

Corollary 2.12. Every 2-local triple derivation on a Cartan factor of type 1 is a triple derivation.

\section{BOUNDEDNESS OF COMPLETELY ADDITIVE MEASURES ON CONTINUOUS $\mathrm{JW}^{*}$-ALGEBRAS}

In this section we shall establish one of the main results of this note, namely a Jordan version of Dorofeev's boundedness theorem (compare [39, Theorem 29.5] or [17, Theorem 1]). The latter states that any completely additive signed measure on the projections of a continuous von Neumann algebra is bounded.

Theorem 3.1 provides the key tool for the proof of Theorem 4.6, which together with Theorem 2.3 leads to the second main conclusion of this note in Theorem 4.7, namely, that a 2-local triple derivation on a continuous $\mathrm{JBW}^{*}$-triple is a triple derivation.

Assume that $M$ is a continuous von Neumann algebra and $\beta: M \rightarrow M$ is a $\mathbb{C}$-linear *-involution (i.e. a *-antiautomorphism of order 2). The subspace $H(M, \beta)$, of all $\beta$-fixed points in $M$, is not, in general, a subalgebra of $M$. However, $H(M, \beta)$ is a weak* closed Jordan *-subalgebra of $M$, whenever the latter is equipped with its natural Jordan product

$$
x \circ y:=\frac{1}{2}(x y+y x) \text {. }
$$

In particular, the self-adjoint part, $H(M, \beta)_{s a}$, of $H(M, \beta)$ is a JBW-subalgebra of $M_{s a}$.

Theorem 3.1. Let $M$ be a continuous von Neumann algebra and let $\beta: M \rightarrow M$ be a $\mathbb{C}$ linear ${ }^{*}$-involution. Let $\Delta: \mathcal{P}(H(M, \beta)) \rightarrow \mathbb{C}$ be a completely additive (complex) measure. Then $\Delta$ is bounded.

The authors do not know if Theorem 3.1 remains valid when $H(M, \beta)$ is replaced by an arbitrary $\mathrm{JBW}^{*}$-algebra containing no summands of type $I_{n}$. See Problem 4.8. However, Theorem 3.1 is sufficient for the purposes of this paper.

We shall show how the arguments in [17] can be adapted to prove the above result. For completeness reasons, we shall present here a draft of the original arguments employed in 
the proof of [17, Theorem 1], making the adjustments, some of which are non-trivial, for the Jordan case. The proof of Theorem 3.1 will occupy us throughout this section.

The following Jordan version of the Bunce-Wright-Mackey-Gleason theorem is an instance of a theorem due to Matveichuk and has been borrowed from [35]

Theorem 3.2. [35, Theorem 1] Let $M$ be a continuous von Neumann algebra and let $\beta$ : $M \rightarrow M$ be a $\mathbb{C}$-linear ${ }^{*}$-involution. Let $\Delta: \mathcal{P}(H(M, \beta)) \rightarrow \mathbb{C}$ be a bounded finitely additive measure. Then there exists a functional $\varphi$ in $H(M, \beta)^{*}$ such that $\Delta(p)=\varphi(p)$, for every $p \in \mathcal{P}(H(M, \beta))$. Furthermore, when $\Delta$ is completely additive the functional $\varphi$ can be assumed to be in $H(M, \beta)_{*}$.

Suppose that $M$ acts on a complex Hilbert space $H$. Following [17], given two projections $p, q \in \mathcal{P}(M)$, the distance between $p$ and $q$ is defined by

$$
d(p, q)=\inf \{\|\xi-\eta\|: \xi \in p(H), \eta \in q(H),\|\xi\|=\|\eta\|=1\} .
$$

Let us take $\xi \in p(H), \eta \in q(H)$ with $\|\xi\|=\|\eta\|=1$. In this case

$$
\|\xi-\eta\| \geq\|\xi-q(\xi)\|-\|q(\xi-\eta)\| \geq\|\xi-q(\xi)\|-\|\xi-\eta\|,
$$

which gives $2\|\xi-\eta\| \geq\|\xi-q(\xi)\|$. Therefore

$$
2\|\xi-\eta\| \geq \inf \{\|\zeta-q(\zeta)\|: \zeta \in p(H),\|\zeta\|=1\},
$$

and thus

$$
d(p, q) \geq \frac{1}{2} \inf \{\|\zeta-q(\zeta)\|: \zeta \in p(H),\|\zeta\|=1\} .
$$

Following standard notation, given two projections $p, q$ in a von Neumann algebra $M$, the symbols $p \vee q$ and $p \wedge q$ will denote the supremum and the infimum of $p$ and $q$ in $M$, respectively. Let $\beta$ be a $\mathbb{C}$-linear *-involution on $M$. It is clear that $\beta(\mathbf{1})=\mathbf{1}$. Furthermore, $\beta(p \vee q)=\beta(p) \vee \beta(q)$ and $\beta(p \wedge q)=\beta(p) \wedge \beta(q)$. So, if $p, q \in H(M, \beta)$, then $p \vee q$ and $p \wedge q$ both belong to $H(M, \beta)$. Having these comments in mind, the arguments in the proof of [17, Lemma 2] can be slightly adapted to obtain:

Lemma 3.3. Let $M$ be a continuous von Neumann algebra and let $\beta: M \rightarrow M$ be a $\mathbb{C}$ linear ${ }^{*}$-involution. Let $\Delta: \mathcal{P}(H(M, \beta)) \rightarrow \mathbb{C}$ be a completely additive measure. Suppose there exists a constant $C>0$ and an increasing sequence $\left(q_{n}\right)$ of projections in $H(M, \beta)$ such that $\left(q_{n}\right) \uparrow \mathbf{1}$ and

$$
\sup \left\{|\Delta(q)|: q \in \mathcal{P}(H(M, \beta)): q \leq q_{n}\right\} \leq C,
$$

for every natural $n$. Then $\Delta$ is bounded.

Proof. Let us observe that $\Delta$ being a completely additive measure implies that for every increasing (respectively, decreasing) sequence $\left(r_{n}\right)$ in $\mathcal{P}(H(M, \beta))$ with $\left(r_{n}\right) \uparrow r$ (respectively, $\left.\left(r_{n}\right) \downarrow r\right)$, where $r \in \mathcal{P}(H(M, \beta))$, then $\Delta\left(r_{n}\right) \rightarrow \Delta(r)$.

We shall show that the set $\{|\Delta(p)|: p \in \mathcal{P}(H(M, \beta))\}$ is bounded. Let us fix $p \in$ $\mathcal{P}(H(M, \beta))$. Since $\left(p_{n}\right)=\left(\mathbf{1}-q_{n}\right) \downarrow 0$ and $p_{n} \wedge(\mathbf{1}-p) \leq p_{n}$, we deduce that $\left(\left|\Delta\left(p_{n}\right)\right|\right)$, 
and $\left(\left|\Delta\left(p_{n} \wedge(\mathbf{1}-p)\right)\right|\right)$ tend to 0 . We can therefore assume that $\left|\Delta\left(p_{n}\right)\right|,\left|\Delta\left(p_{n} \wedge(\mathbf{1}-p)\right)\right| \leq 1$, for each natural $n$.

We claim that for each natural $n$ and every projection $r \in \mathcal{P}(H(M, \beta))$ we have

$$
\left|\Delta\left(r \vee p_{n}\right)\right| \leq 1+C .
$$

Indeed, since $r \vee p_{n} \geq p_{n}$, it follows that $r \vee p_{n}=r \vee p_{n}-p_{n}+p_{n}$ with $r \vee p_{n}-p_{n} \perp p_{n}$. Therefore $\Delta\left(r \vee p_{n}\right)=\Delta\left(r \vee p_{n}-p_{n}\right)+\Delta\left(p_{n}\right)$. Since $p_{n}\left(r \vee p_{n}-p_{n}\right)=0=\left(r \vee p_{n}-p_{n}\right) p_{n}$, we deduce that $r \vee p_{n}-p_{n} \leq \mathbf{1}-p_{n}=q_{n}$. It follows from the assumptions that $\left|\Delta\left(r \vee p_{n}\right)\right| \leq$ $\left|\Delta\left(r \vee p_{n}-p_{n}\right)\right|+\left|\Delta\left(p_{n}\right)\right| \leq C+1$, as desired.

With $p$ as above, let us denote $q=p+(\mathbf{1}-p) \wedge p_{1}$. It is easy to check that $(\mathbf{1}-q) \wedge p_{1}=0$, therefore Remark 1 in [17] proves that $q=r\left(q p_{1} q\right)+q \wedge\left(\mathbf{1}-p_{1}\right)$ with $r\left(q p_{1} q\right) \perp q \wedge\left(\mathbf{1}-p_{1}\right)$. Since $p \perp(\mathbf{1}-p) \wedge p_{1}$, we deduce from the finite additivity of $\Delta$ that $\Delta(q)=\Delta(p)+\Delta((1-$ $\left.p) \wedge p_{1}\right)$ and $\Delta(q)=\Delta\left(r\left(q p_{1} q\right)\right)+\Delta\left(q \wedge\left(\mathbf{1}-p_{1}\right)\right)$, and hence

$$
\begin{gathered}
|\Delta(p)| \leq|\Delta(q)|+\left|\Delta\left((\mathbf{1}-p) \wedge p_{1}\right)\right| \leq\left|\Delta\left(r\left(q p_{1} q\right)\right)\right|+\left|\Delta\left(q \wedge\left(\mathbf{1}-p_{1}\right)\right)\right|+1 \\
\leq\left|\Delta\left(r\left(q p_{1} q\right)\right)\right|+C+1 .
\end{gathered}
$$

The sequence $\left.\left(G_{n}\right)=\left(1_{\left(0,1-\frac{1}{n}\right)}\left(q p_{1} q\right)\right) \subseteq \mathcal{P}(H(M, \beta))\right\}$ grows to the range projection $r\left(q p_{1} q\right)$. We deduce that $\left(\Delta\left(G_{n}\right)\right) \uparrow \Delta\left(r\left(q p_{1} q\right)\right)$, and thus, there exists $n_{1} \in \mathbb{N}$ such that $\left|\Delta\left(G_{n_{1}}\right)-\Delta\left(r\left(q p_{1} q\right)\right)\right|<1$, and consequently,

$$
|\Delta(p)| \leq 2+C+\left|\Delta\left(G_{n_{1}}\right)\right| .
$$

We claim that $G=G_{n_{1}}$ is "separated" from $p_{1}$ in the sense of [17], that is, $d\left(p_{1}, G_{n_{1}}\right)>0$. Considering the von Neumann subalgebra generated by the element $q p_{1} q$ and the functional calculus it is easy to see that $q p_{1} q \leq\left(1-\frac{1}{n_{1}}\right) G+1_{\left[1-\frac{1}{n_{1}}, 1\right]}\left(q p_{1} q\right)$, with $\left(1-\frac{1}{n_{1}}\right) G \perp$ $1_{\left[1-\frac{1}{n_{1}}, 1\right]}\left(q p_{1} q\right)$. Then for each normal state $\varphi \in M_{*}$ with $\varphi(G)=1=\|\varphi\|$, we have $\varphi\left(q p_{1} q\right) \leq 1-\frac{1}{n_{1}}$. Consequently, for each $\xi \in G(H)$ with $\|\xi\|=1$, we have $\left\langle q p_{1} q(\xi) / \xi\right\rangle \leq$ $1-\frac{1}{n_{1}}$. Having in mind that $G \leq r\left(q p_{1} q\right) \leq q$, we deduce that $q(\xi)=\xi$, and hence $\left\langle p_{1}(\xi) / \xi\right\rangle \leq 1-\frac{1}{n_{1}}$, for every $\xi$ as above. This shows that $\left\|\xi-p_{1}(\xi)\right\| \geq \frac{1}{\sqrt{n_{1}}}$, for every $\xi$ satisfying the above conditions. The inequality in (3.1) shows that $d\left(G, p_{1}\right) \geq \frac{1}{2 \sqrt{n_{1}}}$, proving that $G$ is separated from $p_{1}$. Therefore, $d\left(G, p_{n}\right) \geq \frac{1}{2 \sqrt{n_{1}}}$, for every $n \in \mathbb{N}$. Lemma $1(b)$ in [17] shows that

$$
G \vee p_{n} \leq \frac{16}{d\left(G, p_{n}\right)^{2}}\left(G+p_{n}\right) \leq 64 n_{1}\left(G+p_{n}\right),
$$

for every natural $n$. We deduce that $\lim _{n} G \vee p_{n} \leq \lim _{n} 64 n_{1}\left(G+p_{n}\right)=64 n_{1} G$, which implies that $G \vee p_{n} \downarrow G$. We can find $n_{2} \in \mathbb{N}^{\mathbb{N}}$ satisfying $|\Delta(G)| \leq 1+\left|\Delta\left(G \vee p_{n_{2}}\right)\right|$. Combining (3.3) and (3.2) we obtain

$$
|\Delta(p)| \leq 3+C+\left|\Delta\left(G \vee p_{n_{2}}\right)\right| \leq 4+2 C .
$$

The conclusion of the lemma follows from the arbitrariness of $p$. 
The following result for projections in von Neumann algebras is part of the folklore (cf. [17, Lemma 3] or [22, Lemma 6.1.10]. Let us observe that in the latter results the normal state should have been assumed to be faithful). By using the halving lemma for JBW-algebras the same proof holds in the case of $\mathrm{JBW}^{*}$-algebras.

Lemma 3.4. Let $M$ be a continuous von Neumann algebra and let $\beta: M \rightarrow M$ be $a \mathbb{C}$ linear ${ }^{*}$-involution. Suppose $p$ is a projection in $H(M, \beta), \varphi$ is a faithful normal state in $H(M, \beta)_{*}$ and $0<\delta<1$. Then there exists a family of pairwise orthogonal projections $\left(p_{i}\right)_{i=1, \ldots, n}$ in $H(M, \beta)$ satisfying:

(a) $p=\sum_{i=1}^{n} p_{i}$;

(b) $\varphi\left(p_{i}\right) \leq \delta$, for every $i=1, \ldots, n$;

(c) $n \leq 2 / \delta$.

The following result is a crucial point in the proof of the main theorem of this section.

Proposition 3.5. Let $W$ be a JW-algebra containing no finite Type I part. Then $W$ contains a JW-subalgebra B of Type $I_{1}$. Furthermore, if $M$ is a (properly infinite) continuous von Neumann algebra and $\beta: M \rightarrow M$ is a $\mathbb{C}$-linear ${ }^{*}$-involution, then there exists a type $I_{1}$ von Neumann subalgebra $N$ of $M$ satisfying $\beta(N)=N$.

The proof of the above proposition will follow from a technical lemma. First, we recall that a real flip $\alpha$ on $B(H)$ is a *-antiautomorphism of order 2 given by

$$
\alpha(x)=J x^{*} J,
$$

where $J$ is a conjugation on $H$. In this setting

$$
B(H)_{s a}^{\alpha}=\left\{x \in B(H): \alpha(x)=x=x^{*}\right\}
$$

is a Type I JW-algebra factor. Since any two conjugations on the same complex Hilbert space are unitarily equivalent (see [23, Lemma 7.5.6]) all factor JW-algebras arising from a real flip on a fixed Hilbert space are isomorphic.

Lemma 3.6. Let $\alpha$ be a real flip on $B(H)$, where $H$ is a separable and infinite dimensional complex Hilbert space. Then there exists a factor von Neumann algebra $N$ of type $I_{1}$, such that $N$ is an $\alpha$-invariant subalgebra of $B(H)$. In particular, $H(N, \alpha)_{\text {sa }}=\{x \in N$ : $\left.\alpha(x)=x^{*}=x\right\}$ is a finite Type $I_{1} J W$-factor contained in $B(H)_{s a}^{\alpha}$. Moreover, $H(N, \alpha)_{s a}$ is not isomorphic to the self-adjoint part of a von Neumann algebra and the enveloping von Neumann algebra of $H(N, \alpha)_{\text {sa }}$ coincides with $N$.

Proof. Let $\Pi$ be the group of all permutations of natural numbers leaving all but finite integers fixed. $\Pi$ is infinite and countable and so we can suppose that $H=\ell^{2}(\Pi)$. Denote by $\xi_{t}$ an element in $\ell^{2}(\Pi)$ that takes value 1 at $t \in \Pi$ and zero otherwise. Then $\left(\xi_{t}\right)_{t \in \Pi}$ forms an orthonormal basis of $H$. By the remark preceding this lemma, there is no loss of generality in assuming that the real flip $\alpha$ is induced by a conjugation $J$ of the form:

$$
J\left(\sum_{t \in \Pi} \alpha_{t} \xi_{t}\right)=\sum_{t \in \Pi} \overline{\alpha_{t}} \xi_{t},
$$


where $\left(\alpha_{t}\right) \in \ell^{2}(\Pi)$. Let $\mathcal{L}_{G}$ be the (left) group von Neumann algebra generated by the unitaries $u_{t}(t \in \Pi)$, where

$$
u_{t} \xi_{s}:=\xi_{t s}
$$

Since

$$
\begin{gathered}
\alpha\left(u_{t}\right) \xi_{s}=J u_{t}^{*} J \xi_{s}=J u_{t}^{*} \xi_{s}=J u_{t^{-1}} \xi_{s}=\xi_{t^{-1} s}=u_{t^{-1}} \xi_{s}=u_{t}^{*} \xi_{s}, \\
\alpha\left(u_{t}\right)=u_{t}^{*}
\end{gathered}
$$

and consequently, $\mathcal{L}_{G}$ is $\alpha$-invariant, and hence $\alpha\left(\mathcal{L}_{G}\right)=\mathcal{L}_{G}$.

By [29, Example 6.7.7, page 438 and Theorem 6.7.5] $\mathcal{L}_{G}$ is a Type $\mathrm{II}_{1}$ factor (see also [29, Theorem 6.7.2]).

Now, since $\mathcal{L}_{G}$ is a continuous von Neumann factor, we conclude, by Theorem 1.5.2 in [5], that the algebra $H\left(\mathcal{L}_{G}, \alpha\right)_{s a}:=\left\{x \in \mathcal{L}_{G}: \alpha(x)=x=x^{*}\right\}$ is a continuous JWalgebra factor which is not isomorphic to the self-adjoint part of a von Neumann algebra and the enveloping von Neumann algebra of $H\left(\mathcal{L}_{G}, \alpha\right)_{s a}$ coincides with $\mathcal{L}_{G}$. Moreover, Theorem 1.3.2 in [5] implies that $H\left(\mathcal{L}_{G}, \alpha\right)$ is finite.

Proof of Proposition 3.5. Let us suppose first that $W$ is infinite and homogeneous Type $\mathrm{I}_{n}$, where $n$ is an infinite cardinal number. Then, according to the structure theory (see [23, Definition 5.3.3(ii)]), we can find an infinite system $\left(p_{j}\right)_{j \in \Lambda}$ of mutually orthogonal abelian projections such that $\sum_{j} p_{j}=1$, the central support projection of each $p_{j}$ coincides with the unit of $W$ and $\operatorname{card}(\Lambda)=n$. We can also conclude that the $p_{j}$ 's are mutually exchangeable by a symmetry (compare [23, Lemma 5.3.2]). Clearly, we can restrict to a countable subfamily. Then, there is a unital JW-subalgebra of $W$ containing $\left(p_{j}\right)$ that is isomorphic to $B(H)_{s a}^{\alpha}$, where $\alpha$ is a real flip and $H$ has infinite countable dimension (see [23, Theorem 7.6.3 $(i i i) \Leftrightarrow$ $(i v)])$. The desired conclusion follows, in this case, from Lemma 3.6.

By [41, Theorem 16] (alternatively, [23, Theorem 5.3.5]) any properly infinite Type I JW-algebra $W$ can be decomposed into a direct sum of infinite homogeneous ones. We can obtain the desired finite type $\mathrm{II}_{1}$ continuous $\mathrm{JW}$-subalgebra $B$ by taking the sum of all type $\mathrm{II}_{1} \mathrm{JW}$-subfactors given by Lemma 3.6 in the corresponding homogeneous summand. Actually it is enough to consider a non-zero type $\mathrm{II}_{1} \mathrm{JW}$-subfactor in any of the corresponding homogeneous summands.

We assume now that $W$ contains no type I part. Let $p$ be a non-zero projection in $W$. If $p$ is modular then $B=\{p, W, p\}$ is a $\mathrm{JW}$-algebra of type $\mathrm{II}_{1}$, which proves the desired statement. If $p$ is not modular, then $\{p, W, p\}$ contains a copy of $B(H)_{s a}^{\alpha}$, where $H$ is separable and infinite dimensional, and $\alpha$ is a real flip (see Theorem 7.6.3 $(i) \Leftrightarrow(i v)$ in [23]). Lemma 3.6 implies the existence of a type $\mathrm{II}_{1} \mathrm{JW}$-subfactor of $B(H)_{s a}^{\alpha}$. This finishes the proof of the first statement in Proposition 3.5.

We consider now the second statement in the proposition. Let $M$ be a continuous von Neumann algebra and suppose $\beta: M \rightarrow M$ is a $\mathbb{C}$-linear ${ }^{*}$-involution. We may assume, without loss of generality, that the type $\mathrm{II}_{1}$ part of $M$ is zero. We consider the JW-algebra $H(M, \beta)_{s a}=\left\{a \in M: \beta(a)=a=a^{*}\right\}$.

We claim that $H(M, \beta)_{s a}$ contains a central projection which is not modular. Let $z$ be a central projection in $H(M, \beta)_{s a}$. If $z$ is not modular the claim is obvious, otherwise 
$z H(M, \beta)_{s a} z$ is modular. Let $R(M, \beta)=\left\{x \in M: \beta(x)=x^{*}\right\}$. Clearly, $R(M, \beta)$ is a real von Neumann algebra and $H(M, \beta)_{s a}=\left\{x \in R(M, \beta): x=x^{*}\right\}$ coincides with the hermitian part of $R(M, \beta)$. We also have $M=R(M, \beta)+i R(M, \beta)$, via $x=\left(x+\beta\left(x^{*}\right)\right) / 2+i(x-$ $\left.\beta\left(x^{*}\right)\right) / 2 i$ ). We observe that $z$ is a projection in $M$ with $\beta(z)=z^{*}=z, z M z$ is $\beta$-invariant, and $z H(M, \beta)_{s a} z=H(z M z, \beta)_{s a}=R(z M z, \beta)_{s a}$. We deduce from Proposition 1.3 in [1] that $R(z M z, \beta)$ is finite. Theorem 2.2 in [1] implies that $z M z=R(z M z, \beta)+i R(z M z, \beta)$ (and hence $z$ ) is finite in $M$. Let $c(z)$ denote the central support projection of $z$ in $M$, that is, $c(z)$ is the smallest central projection in $M$ majorizing $z$. Since $\beta$ is a $\mathbb{C}$-linear *-involution, we deduce that $\beta(c(z))=c(\beta(z))=c(z)$, and thus $c(z)$ lies in $H(M, \beta)_{s a}$. Since the type $\mathrm{II}_{1}$ part of $M$ is zero and $M$ is continuous, we deduce that $c(z)$ must be an infinite central projection in $M$ (compare [40, Definition V.1.17]). Thus $c(z)$ must be a non-modular central projection in $H(M, \beta)_{s a}$, which proves the claim. Indeed, if $c(z) H(M, \beta)_{s a}$ were modular, then as shown above, $c(z) M$ would be finite.

Finally, let $p$ be a non-modular central projection in $H(M, \beta)_{s a}$. A new application of [23, Theorem 7.6.3 $(i) \Leftrightarrow(i v)]$ implies that $\left\{p, H(M, \beta)_{s a}, p\right\}=U_{p}\left(H(M, \beta)_{s a}\right)$ contains a copy of $B(H)_{s a}^{\alpha}$, where $H$ is separable and infinite dimensional, and $\alpha$ is a real flip. By Lemma 3.6 there exists a von Neumann algebra $N$ of type $\mathrm{II}_{1}$ such that $N$ is an $\alpha$-invariant von Neumann subalgebra of $B(H), H(N, \alpha)_{s a}$ is a JBW-subalgebra of $H(M, \beta)_{s a}$ and the enveloping von Neumann algebra of $H(N, \alpha)_{s a}$ coincides with $N$. Clearly $N$ is a subalgebra of $M$. Since every $x \in H(N, \alpha)_{s a}$ satisfies $\beta(x)=x=x^{*}$ and $\beta$ is a $\mathbb{C}$-linear ${ }^{*}$-involution, the enveloping von Neumann algebra of $H(N, \alpha)_{s a}$, namely $N$, must be $\beta$-invariant, which concludes the proof.

The remaining results in this section are appropriate adaptations of the corresponding lemmas in [17] and [22, 6.1$]$, they are included here for completeness reasons.

Let us observe a simple property.

Remark 3.7. Let $M$ be a von Neumann algebra, let $\beta$ be a $\mathbb{C}$-linear ${ }^{*}$-involution on $M$, and let $\Delta: \mathcal{P}(H(M, \beta)) \rightarrow \mathbb{C}$ be a completely additive measure. Suppose we can decompose $M$ as finite direct sum of mutually orthogonal $\beta$-invariant von Neumann subalgebras $M_{1}, \ldots, M_{k}$, that is $M=M_{1} \oplus^{\infty} \ldots \oplus^{\infty} M_{k}$ with $\beta\left(M_{j}\right)=M_{j}$, for every $j$. Then $\Delta$ is bounded if and only if $\left.\Delta\right|_{\mathcal{P}\left(H\left(M_{j}, \beta\right)\right)}: \mathcal{P}\left(H\left(M_{j}, \beta\right)\right) \rightarrow \mathbb{C}$ is bounded for every $j=1, \ldots, k$.

Let us briefly recall some basic notions on $\sigma$-finite projections in $\mathrm{JBW}^{*}$-algebras. As in the setting of von Neumann algebras, a $\mathrm{JBW}^{*}$-algebra $M$ is said to be $\sigma$-finite if every family of mutually orthogonal non-zero projections in $M$ is at most countable. A projection $p$ in $M$ is called $\sigma$-finite if the $\mathrm{JBW}^{*}$-algebra $U_{p}(M)$ is $\sigma$-finite, where $U_{p}$ is the operator on $M$ given by $U_{p}(x)=\left\{p, x^{*}, p\right\}=2(p \circ x) \circ p-p \circ x$. A projection $p$ in $M$ is $\sigma$-finite if and only if it is the support projection of a normal state in $M_{*}$ (cf. [19, Theorem 3.2]). The supremum of countably many $\sigma$-finite projections is again $\sigma$-finite, and every projection in a JBW ${ }^{*}$-algebra can be written as a sum of mutually orthogonal $\sigma$-finite projections (see [19, Theorem 3.4]). These facts can be derived from [19] and are explicitly developed in [7].

The following two results will be applied in several arguments (compare [17, Lemma 4]). 
Proposition 3.8. Let $M$ be a continuous von Neumann algebra and let $\beta: M \rightarrow M$ be a $\mathbb{C}$-linear ${ }^{*}$-involution. Suppose that $\Delta: \mathcal{P}(H(M, \beta)) \rightarrow \mathbb{C}$ is an unbounded completely additive measure. Then there exists a $\sigma$-finite projection $p \in H(M, \beta)$ such that $\left.\Delta\right|_{\mathcal{P}(H(p M p, \beta))}$ is unbounded.

Proof. Since $\Delta$ is unbounded, there exists a sequence $\left(q_{n}\right)$ in $\mathcal{P}(H(M, \beta))$ satisfying that $\lim _{n \rightarrow \infty}\left|\Delta\left(q_{n}\right)\right|=\infty$. Each $q_{n}$ can be written as the sum of a family of mutually orthogonal $\sigma$-finite projections in $H(M, \beta)$ (compare [19, Theorem $3.4(i i)]$ ). Therefore, there exists a family $\left(p_{\lambda}^{(n)}\right)_{\lambda \in \Lambda_{n}}$ of mutually orthogonal $\sigma$-finite projections in $H(M, \beta)$ such that $q_{n}=$ $\sum_{\lambda \in \Lambda_{n}} p_{\lambda}^{n}$. By the complete additivity of $\Delta$, there exists a finite subset $F_{n} \subseteq \Lambda_{n}$ such that

$$
\left|\Delta\left(q_{n}\right)-\Delta\left(\sum_{\lambda \in F_{n}} p_{\lambda}^{(n)}\right)\right|<\frac{1}{n} .
$$

Clearly, $p_{n}=\sum_{\lambda \in F_{n}} p_{\lambda}^{n}$ is a $\sigma$-finite projection in $\mathcal{P}(H(M, \beta))$ and $\lim _{n \rightarrow \infty}\left|\Delta\left(p_{n}\right)\right|=\infty$. Let $p=\bigvee_{n} p_{n} \in \mathcal{P}(H(M, \beta))$. Since the supremum of countably many $\sigma$-finite projections is again $\sigma$-finite (compare [19, Theorem $3.4(i)]$ ), the projection $p$ is $\sigma$-finite, and obviously, $\left.\Delta\right|_{\mathcal{P}(H(p M p, \beta))}$ is unbounded, which finishes the proof.

Proposition 3.9. Let $M$ be a von Neumann algebra of type $I_{1}, I_{\infty}$ or III, and let $\beta$ a $\mathbb{C}$-linear ${ }^{*}$-involution on $M$. Suppose $\Delta: \mathcal{P}(H(M, \beta)) \rightarrow \mathbb{C}$ is a completely additive unbounded measure. Then there exists a projection $p_{0} \in \mathcal{P}(H(M, \beta))$ such that $p_{0} M p_{0}$ is $\sigma$-finite and of type $I I_{1}, I I_{\infty}$ or III, and the measure $\Delta_{0}=\left.\Delta\right|_{\mathcal{P}\left(H\left(p_{0} M p_{0}, \beta\right)\right)}: \mathcal{P}\left(H\left(p_{0} M p_{0}, \beta\right)\right) \rightarrow \mathbb{C}$ satisfies the following property

$$
\begin{gathered}
\text { for each } p \in \mathcal{P}\left(H\left(p_{0} M p_{0}, \beta\right)\right) \text { with }\left|\Delta_{0}(p)\right|>1 \text { the measure } \\
\left.\Delta_{0}\right|_{\mathcal{P}\left(H\left(\left(p_{0}-p\right) M\left(p_{0}-p\right), \beta\right)\right)}: \mathcal{P}\left(H\left(\left(p_{0}-p\right) M\left(p_{0}-p\right), \beta\right)\right) \rightarrow \mathbb{C} \text { is bounded. }
\end{gathered}
$$

Proof. If the pair $(H(M, \beta), \Delta)$ satisfies the desired property then the proof is concluded by taking $p_{0}=1$. Otherwise, there exists a projection $p_{1} \in \mathcal{P}(H(M, \beta))$ with $\left|\Delta\left(p_{1}\right)\right|>1$ satisfying that the measure $\left.\Delta\right|_{\mathcal{P}\left(H\left(\left(\mathbf{1}-p_{1}\right) M\left(\mathbf{1}-p_{1}\right), \beta\right)\right)}: \mathcal{P}\left(H\left(\left(\mathbf{1}-p_{1}\right) M\left(\mathbf{1}-p_{1}\right), \beta\right)\right) \rightarrow \mathbb{C}$ is unbounded. Since $\left(\mathbf{1}-p_{1}\right) M\left(\mathbf{1}-p_{1}\right)$ doesn't contain type I part, we can decompose $\left(\mathbf{1}-p_{1}\right) M\left(\mathbf{1}-p_{1}\right)$ as a direct sum of von Neumann subalgebras of type $\mathrm{II}_{1}, \mathrm{II}_{\infty}$ or III. We also observe that each summand in the above decomposition must be $\beta$-invariant. Therefore, by Remark 3.7, there exists a subprojection $q_{1} \leq \mathbf{1}-p_{1}$ such that $q_{1} M q_{1}$ is of type $\mathrm{II}_{1}, \mathrm{II}_{\infty}$ or III and $\left.\Delta\right|_{\mathcal{P}\left(H\left(q_{1} M q_{1}, \beta\right)\right)}$ is unbounded.

If the pair $\left(H\left(q_{1} M q_{1}, \beta\right),\left.\Delta\right|_{\mathcal{P}\left(H\left(q_{1} M q_{1}, \beta\right)\right)}\right)$ satisfies property (3.4) we obtain the desired statement. Otherwise, applying the above argument, there exists $p_{2} \leq q_{1}$ in $H\left(q_{1} M q_{1}, \beta\right)$ such that $\left|\Delta\left(p_{2}\right)\right|>1$ and $\left.\Delta\right|_{\mathcal{P}\left(H\left(\left(q_{1}-p_{2}\right) M\left(q_{1}-p_{2}\right), \beta\right)\right)}: \mathcal{P}\left(H\left(\left(q_{1}-p_{2}\right) M\left(q_{1}-p_{2}\right), \beta\right)\right) \rightarrow \mathbb{C}$ is unbounded. Thus, there exists $q_{2} \leq q_{1}-p_{2}$ such that $q_{2} M q_{2}$ is of type $\mathrm{II}_{1}, \mathrm{II}_{\infty}$ or III and $\left.\Delta\right|_{\mathcal{P}\left(H\left(q_{2} M q_{2}, \beta\right)\right)}$ is unbounded. 
By repeating the above arguments, we find a pair $\left(q_{n} M q_{n},\left.\Delta\right|_{\mathcal{P}\left(H\left(q_{n} M q_{n}, \beta\right)\right)}\right)$ (with $\beta\left(q_{n}\right)=$ $q_{n}$, for every $n \in \mathbb{N}$ ) satisfying the desired statement, or there exists an infinite sequence $\left(p_{n}\right)$ of mutually orthogonal $\beta$-symmetric projections in $M$ satisfying $\left|\Delta\left(p_{n}\right)\right|>1$, for every natural $n$, which contradicts the complete additivity of $\Delta$.

Henceforth, up to and including Lemma 3.14, $M$ will denote a $\sigma$-finite von Neumann algebra of type $\mathrm{II}_{1}, \mathrm{II}_{\infty}$ or III, $\beta$ a $\mathbb{C}$-linear *-involution on $M$, and $N$ a type $\mathrm{II}_{1}$ von Neumann subalgebra of $M$ satisfying $\beta(N)=N$ (compare Proposition 3.5). We observe that $H(N, \beta)$ is a $\mathrm{JBW}^{*}$-subalgebra of $H(M, \beta)$. From now on, $\tau$ will stand for a faithful normal norm-one finite trace on $N$, whose restriction to $H(N, \beta)$ will be also denoted by $\tau$.

First, we recall some facts about the strong* topology. For each normal positive functional $\varphi$ in the predual of a von Neumann algebra $M$, the mapping

$$
x \mapsto\|x\|_{\varphi}=\left(\varphi\left(\frac{x x^{*}+x^{*} x}{2}\right)\right)^{\frac{1}{2}} \quad(x \in M)
$$

defines a prehilbertian seminorm on $M$. The strong* topology of $M$, denoted by $S^{*}\left(M, M_{*}\right)$, is the locally convex topology on $M$ defined by all the seminorms $\|.\|_{\varphi}$, where $\varphi$ runs in the set of all positive functionals in $M_{*}$

Lemma 3.10. Let $\Delta: \mathcal{P}(H(M, \beta)) \rightarrow \mathbb{C}$ be a completely additive unbounded measure. Suppose that the pair $(H(M, \beta), \Delta)$ satisfies property (3.4) in Lemma 3.9. Let $N$ a type II von Neumann subalgebra of $M$ satisfying $\beta(N)=N$, and let $\tau$ denote the unital normal faithful finite trace on $N$. Then there exist a positive constant $K$ and $0<\delta<1$ satisfying the following property:

For each $q \in H(N, \beta)$ with $\tau(q) \leq \delta$, we have $\sup \{|\Delta(p)|: p \in H(M, \beta), p \leq q\} \leq K$.

Proof. (compare [17, Lemma 5]) Arguing by reduction to the absurd, we suppose that the desired property does not hold. Then there exists a sequence $\left(q_{n}\right)$ in $H(N, \beta)$ such that $\left|\tau\left(q_{n}\right)\right| \leq \frac{1}{2^{n}}$ and

$$
\sup \left\{|\Delta(p)|: p \in H(M, \beta), p \leq q_{n}\right\}>n,
$$

for every natural $n$. Set $G_{n}:=\vee_{k=n}^{\infty} q_{k}$. Since every $q_{n}$ is $\beta$-symmetric, we deduce that $G_{n}$ also is $\beta$-symmetric for all $n \in \mathbb{N}$ (i.e., $\left(G_{n}\right) \subset H(N, \beta)$ ). Considering strong*-limits of growing sequences, we deduce that $\tau\left(G_{n}\right) \leq \sum_{k=n}^{\infty} \tau\left(q_{k}\right) \leq \sum_{k=n}^{\infty} \frac{1}{2^{k}}$, which implies, by the faithfulness of $\tau$ on $N$, that $\left(G_{n}\right) \searrow 0$ in the strong*-topology of $N$ (and also in the strong*-topology of $M$ ). Since $G_{n} \geq q_{n}$ and $G_{n} \downarrow 0$, we have for every $m \geq n$,

$$
\sup \left\{|\Delta(p)| ; p \leq G_{n}\right\} \geq \sup \left\{|\Delta(p)| ; p \leq G_{m}\right\} \geq \sup \left\{|\Delta(p)| ; p \leq q_{n}\right\}>m,
$$

and $\left.\Delta\right|_{\mathcal{P}\left(H\left(G_{n} M G_{n}, \beta\right)\right)}$ is unbounded. Since the pair $(H(M, \beta), \Delta)$ satisfies property (3.4), we deduce that $\sup \left\{|\Delta(p)|: p \in H(M, \beta), p \leq \mathbf{1}-G_{n}\right\} \leq 1$, for every natural $n$. Since $1-G_{n} \nearrow 1$ in the strong*-topology, Lemma 3.3 implies that $\Delta$ is bounded, which is impossible. 
The automatic boundedness of completely additive measure on $\mathcal{P}(H(M, \beta))$ actually relies on the appropriate Jordan version of the Mackey-Gleason theorem stated in Theorem 3.2 .

Lemma 3.11. Let $\Delta: \mathcal{P}(H(M, \beta)) \rightarrow \mathbb{C}$ be a completely additive unbounded measure. Suppose that the pair $(H(M, \beta), \Delta)$ satisfies the property (3.4) in Lemma 3.9. Let $N$ a type $I I_{1}$ von Neumann subalgebra of $M$ satisfying $\beta(N)=N$ and let $\tau$ be the unital faithful normal trace on $N$. Then there exists a positive constant $C_{0}$ satisfying that if $q \in \mathcal{P}(H(N, \beta))$ and $\left.\Delta\right|_{\mathcal{P}(H(q M q, \beta))}$ is bounded then

$$
\sup \{|\Delta(p)|: p \in \mathcal{P}(H(q M q, \beta))\} \leq C_{0} .
$$

Proof. The proof given in [17, Lemma 6] or in [22, Lemma 6.1.13] remains valid here when we replace [17, Lemma 5] with our previous Lemma 3.10 and the Bunce-Wright-MackeyGleason theorem [10] with Theorem 3.2. We include some details for completeness reasons.

Let $C_{0}=\frac{32 K}{\delta}$, where $K$ and $\delta$ are given by Lemma 3.10. Take a projection $q$ in $H(N, \beta)$ with $\left.\Delta\right|_{\mathcal{P}(H(q M q, \beta))}$ bounded. Theorem 3.2 implies the existence of a (normal) continuous linear functional $\varphi: H(q M q, \beta) \rightarrow \mathbb{C}$ such that $\varphi(p)=\Delta(p)$, for every $p \in \mathcal{P}(H(q M q, \beta))$. By Lemma 3.4 there exists a family of pairwise orthogonal projections $\left(q_{i}\right)_{i=1, \ldots, n}$ in $H(N, \beta)$ such that $q=\sum_{i=1}^{n} q_{i}, \tau\left(q_{i}\right) \leq \frac{\delta}{2}$, for every $i=1, \ldots, n$, and $n \leq 4 / \delta$.

Let us pick an arbitrary projection $p \in H(q M q, \beta)$. We need to show that $|\Delta(p)| \leq C_{0}$. If we write $p=\sum_{i, j=1}^{n}\left\{q_{i}, p, q_{j}\right\}=\sum_{i, j=1}^{n} \frac{1}{2}\left(q_{i} p q_{j}+q_{j} p q_{i}\right)$, we observe that, for $i \neq j, q_{i}+q_{j}$ is a projection in $H(N, \beta)$ and $\tau\left(q_{i}+q_{j}\right) \leq \delta$. Lemma 3.10 implies that

$$
\sup \left\{|\Delta(r)|: r \in \mathcal{P}(H(M, \beta)), r \leq q_{i}+q_{j}\right\} \leq K
$$

and hence that

$$
\sup \left\{|\varphi(r)|: r \in \mathcal{P}(H(M, \beta)), r \leq q_{i}+q_{j}\right\} \leq K .
$$

Considering spectral resolutions, we deduce that $\sup \left\{|\varphi(a)|: a \in H\left(\left(q_{i}+q_{j}\right) M\left(q_{i}+\right.\right.\right.$ $\left.\left.\left.q_{j}\right), \beta\right),\|a\| \leq 1\right\} \leq 2 K$. Similarly, $\sup \left\{|\varphi(a)|: a \in H\left(q_{j} M q_{j}, \beta\right),\|a\| \leq 1\right\} \leq 2 K$, for every $j=1, \ldots, n$. Therefore, having in mind that $q_{i} p q_{j}+q_{j} p q_{i}$ lies in $H\left(\left(q_{i}+q_{j}\right) M\left(q_{i}+\right.\right.$ $\left.\left.q_{j}\right), \beta\right)$, we deduce that

$$
|\Delta(p)|=|\varphi(p)| \leq \sum_{i, j=1}^{n} \frac{1}{2}\left|\varphi\left(q_{i} p q_{j}+q_{j} p q_{i}\right)\right| \leq n^{2} 2 K \leq \frac{16}{\delta^{2}} 2 K=C_{0} .
$$

In a similar fashion, replacing [17, Lemmas 2 and 6] with Lemmas 3.3 and 3.11, respectively, the proof of [17, Lemma 7] holds to prove the following result.

Lemma 3.12. Let $\Delta: \mathcal{P}(H(M, \beta)) \rightarrow \mathbb{C}$ be a completely additive unbounded measure, where $H(M, \beta)$ is a $\sigma$-finite $J B W^{*}$-algebra. Suppose that the pair $(H(M, \beta), \Delta)$ satisfies the property (3.4) in Lemma 3.9. Let $N$ a type $I_{1}$ von Neumann subalgebra of $M$ satisfying $\beta(N)=N$. Then there exists a projection $q_{0}$ in $H(N, \beta)$ satisfying the following properties: (a) $\left.\Delta\right|_{\mathcal{P}\left(H\left(q_{0} M q_{0}, \beta\right)\right)}$ is bounded; 
(b) if $q \in \mathcal{P}(H(N, \beta)), q \gtreqless q_{0}$ then $\left.\Delta\right|_{\mathcal{P}(H(q M q, \beta))}$ is unbounded.

Proof. Let $\mathcal{B}$ denote the set of all families $\left(q_{i}\right)_{i \in I}$ of mutually orthogonal projections in $H(N, \beta)$ such that for each finite subset $F \subset I$, the projection $q_{F}:=\sum_{i \in F} q_{i}$ satisfies that $\left.\Delta\right|_{\mathcal{P}\left(H\left(q_{F} M q_{F}, \beta\right)\right)}$ is bounded. The set $\mathcal{B}$ is an inductive set when it is equipped with the order given by inclusion (by Proposition 3.9, $\mathcal{B} \neq \emptyset$ ). By Zorn's lemma there exists a maximal element $\left(q_{i}^{0}\right)_{I} \in \mathcal{B}$. The set $\mathrm{I}$ is at most countable because $H(M, \beta)$ is $\sigma$-finite. We claim that the projection $q_{0}=\sum_{i \in I} q_{i}^{0} \in H(N, \beta)$ satisfies the desired property. Indeed, defining $q_{n}:=\sum_{i=1}^{n} q_{i}^{0}$, we have $q_{n} \nearrow q_{0}$. Since $\left(q_{i}^{0}\right)_{I} \in \mathcal{B}$, the measure $\left.\Delta\right|_{\mathcal{P}\left(H\left(q_{n} M q_{n}, \beta\right)\right)}$ is bounded for every $n$. Lemma 3.11 implies the existence of a constant $C_{0}>0$ such that $\sup \left\{|\Delta(p)|: p \in H(M, \beta), p \leq q_{n}\right\} \leq C_{0}$, for every natural $n$. Lemma 3.3 proves that $\left.\Delta\right|_{\mathcal{P}\left(H\left(q_{0} M q_{0}, \beta\right)\right)}$ is bounded.

Finally, the second property follows from the maximality of the element $\left(q_{i}^{0}\right)_{I} \in \mathcal{B}$.

We shall see now that the arguments in the proof of [22, Lemma 6.1.15] are also valid in the Jordan setting. Actually, the proof follows the arguments we gave in Lemma 3.10.

Lemma 3.13. Let $\Delta: \mathcal{P}(H(M, \beta)) \rightarrow \mathbb{C}$ be a completely additive unbounded measure. Suppose that the pair $(H(M, \beta), \Delta)$ satisfies the property (3.4) in Lemma 3.9. Let us assume that $H(M, \beta)$ is $\sigma$-finite and let $\varphi$ be a faithful normal state on $H(M, \beta)$. Then there exists a projection $p_{0}$ in $H(M, \beta)$ and $\delta>0$ such that $\left.\Delta\right|_{\mathcal{P}\left(H\left(p_{0} M p_{0}, \beta\right)\right)}$ is unbounded and the following property holds:

$$
\text { If } p \in \mathcal{P}(H(M, \beta)), p \leq p_{0} \text { and } \varphi(p) \leq \delta \text {, then }\left.\Delta\right|_{\mathcal{P}(H(p M p, \beta))} \text { is bounded. }
$$

Proof. If the desired property holds for $p_{0}=1$ and some $\delta$, then the Lemma is proved. Otherwise, there exists a projection $p_{1}$ in $\mathcal{P}(H(M, \beta))$ such that $\varphi\left(p_{1}\right) \leq \frac{1}{2}$ and $\left.\Delta\right|_{\mathcal{P}\left(H\left(p_{1} M p_{1}, \beta\right)\right)}$ is unbounded. If $p_{1}$ satisfies the desired property the statement is proved. If that is not the case, there exists a projection $p_{2}$ in $\mathcal{P}(H(M, \beta))$ such that $p_{2} \leq p_{1}, \varphi\left(p_{2}\right) \leq \frac{1}{3}$ and $\left.\Delta\right|_{\mathcal{P}\left(H\left(p_{2} M p_{2}, \beta\right)\right)}$ is unbounded. Repeating the above argument, we obtain the desired conclusion for a suitable projection, or there exists a decreasing sequence of projections $\left(p_{n}\right)$ in $H(M, \beta)$ satisfying $\varphi\left(p_{n}\right) \leq \frac{1}{n}$ and $\left.\Delta\right|_{\mathcal{P}\left(H\left(p_{n} M p_{n}, \beta\right)\right)}$ is unbounded. The faithfulness of $\varphi$ implies that $p_{n} \searrow 0$ in the strong*-topology.

Since the pair $(H(M, \beta), \Delta)$ satisfies property (3.4) in Lemma 3.9, we conclude that

$$
\sup \left\{|\Delta(p)|: p \in \mathcal{P}(H(M, \beta)), p \leq 1-p_{n}\right\} \leq 1,
$$

for all $n$. Recalling that $\mathbf{1}-p_{n} \nearrow \mathbf{1}$ in the strong*-topology, Lemma 3.3 implies that $\Delta$ is bounded, which contradicts the hypothesis of the lemma.

Lemma 3.14. Let $\Delta: \mathcal{P}(H(M, \beta)) \rightarrow \mathbb{C}$ be a completely additive unbounded measure. Let $p_{0}$ be a projection in $H(M, \beta)$ satisfying that $\left.\Delta\right|_{\mathcal{P}\left(H\left(p_{0} M p_{0}, \beta\right)\right)}$ and $\left.\Delta\right|_{\mathcal{P}\left(H\left(\left(1-p_{0}\right) M\left(1-p_{0}\right), \beta\right)\right)}$ are bounded. Let $K_{n} \rightarrow \infty$. Then for each natural $n$, there exists a projection $q=q_{n} \in$ $H(M, \beta)$ such that $|\Delta(q)|>K_{n}$ and $d\left(q, p_{0}\right) \geq \frac{1}{8}$.

Proof. (compare [17, Lemma 9]) Let us take $C>0$ satisfying $\sup \{|\Delta(p)|: p \in H(M, \beta), p \leq$ $p_{0}$ or $\left.p \leq 1-p_{0}\right\} \leq C$. Given $n$, by the unboundedness of $\Delta$, we can find a projection $p$ in 
$H(M, \beta)$ such that $|\Delta(p)|>2 K_{n}+6 C$. The projection $r=p+(\mathbf{1}-p) \wedge p_{0} \in H(M, \beta)$ satisfies $(\mathbf{1}-r) \wedge p=0$ and $|\Delta(r)| \geq|\Delta(p)|-\left|\Delta\left((\mathbf{1}-p) \wedge p_{0}\right)\right|>2 K_{n}+5 C$. By [17, Remark 1] we have

$$
r=r\left(r p_{0} r\right)+r \wedge\left(\mathbf{1}-p_{0}\right)
$$

in $M$, as well as in $H(M, \beta)$. The proof of [17, Lemma 9] shows that taking $r_{1}=1_{\left(0, \frac{1}{2}\right]}\left(r p_{0} r\right) \in$ $H(M, \beta)$ and $r_{2}=1_{\left(\frac{1}{2}, 1\right]}\left(r p_{0} r\right) \in H(M, \beta)$ we have $d\left(r_{1}, p_{0}\right) \geq \frac{1}{2}$ and $r_{1}+r_{2}=r-r \wedge(1-$ $\left.p_{0}\right)$. It is further seen that for $r_{2}^{\prime}=r_{2} \vee\left(\mathbf{1}-p_{0}\right)-r_{2} \in H(M, \beta)$ the inequality $d\left(r_{2}^{\prime}, p_{0}\right) \geq \frac{1}{8}$ holds.

It is also clear that $r_{1} \perp r_{2}$, and since $r-r \wedge\left(\mathbf{1}-p_{0}\right) \perp r \wedge\left(\mathbf{1}-p_{0}\right)$. Therefore

$$
\begin{gathered}
\left|\Delta\left(r_{1}\right)\right|+\left|\Delta\left(r_{2}\right)\right| \geq\left|\Delta\left(r_{1}\right)+\Delta\left(r_{2}\right)\right|=\left|\Delta\left(r_{1}+r_{2}\right)\right|=\left|\Delta\left(r-r \wedge\left(1-p_{0}\right)\right)\right| \\
\quad=\left|\Delta(r)-\Delta\left(r \wedge\left(\mathbf{1}-p_{0}\right)\right)\right| \geq|\Delta(r)|-\left|\Delta\left(r \wedge\left(\mathbf{1}-p_{0}\right)\right)\right|>2 K_{n}+4 C .
\end{gathered}
$$

It follows that $\left|\Delta\left(r_{1}\right)\right|>K_{n}+2 C$ or $\left|\Delta\left(r_{2}\right)\right|>K_{n}+2 C$. In the first case the projection $q=$ $r_{1}$ satisfies the desired statement; otherwise, the projection $q=r_{2}^{\prime}$ satisfies the conclusion of the lemma. Indeed,

$$
\left|\Delta\left(r_{2} \vee\left(\mathbf{1}-p_{0}\right)\right)\right| \leq\left|\Delta\left(p_{0}\right)\right|+\left|\Delta\left(r_{2} \vee\left(\mathbf{1}-p_{0}\right)-\left(\mathbf{1}-p_{0}\right)\right)\right| \leq 2 C,
$$

because $\left(r_{2} \vee\left(\mathbf{1}-p_{0}\right)-\left(\mathbf{1}-p_{0}\right)\right) \perp\left(\mathbf{1}-p_{0}\right)$. Thus, we get

$$
|\Delta(q)|=\left|\Delta\left(r_{2}^{\prime}\right)\right| \geq\left|\Delta\left(r_{2}\right)\right|-\left|\Delta\left(r_{2} \vee\left(1-p_{0}\right)\right)\right|>K_{n} .
$$

We complete now the proof of our Jordan version of Dorofeev's theorem. The arguments are based on appropriate Jordan adaptations of the proofs in [17, Theorem 1] and [22, Theorem 6.1.16].

Proof of Theorem 3.1. Arguing by contradiction, we shall assume that $\Delta: \mathcal{P}(H(M, \beta)) \rightarrow$ $\mathbb{C}$ is an unbounded completely additive measure. By Proposition 3.8 there exists a $\sigma$-finite projection $p \in H(M, \beta)$ such that $\left.\Delta\right|_{\mathcal{P}(H(p M p, \beta))}$ is unbounded.

We can therefore assume that $H(M, \beta)$ is $\sigma$-finite. Let $\varphi$ be a faithful normal state on $H(M, \beta)$. Furthermore, by Remark 3.7, we can also assume that $M$ is of type $\mathrm{II}_{1}, \mathrm{II}_{\infty}$ or III.

Having in mind Proposition 3.9, we can assume that the pair $(H(M, \beta), \Delta)$ satisfies property (3.4) for $p_{0}=1$ in that proposition (otherwise we replace $M$ with $p_{0} M p_{0}$ ). Applying Lemma 3.13, we may assume that $\Delta$ satisfies property (3.6) for $p_{0}=\mathbf{1}$, the faithful normal state $\varphi$ fixed in the above paragraph, and a suitable $\delta>0$. By Proposition 3.5 there exists a type $\mathrm{II}_{1}$ subalgebra $N$ of $M$ such that $\beta(N)=N$.

Let $q_{0}$ be the projection in $H(N, \beta)$ given by Lemma 3.12, that is, $q_{0}$ satisfies the following properties:

(a) $\left.\Delta\right|_{\mathcal{P}\left(H\left(q_{0} M q_{0}, \beta\right)\right)}$ is bounded;

(b) if $q \in \mathcal{P}(H(N, \beta)), q \gtreqless q_{0}$ then $\left.\Delta\right|_{\mathcal{P}(H(q M q, \beta))}$ is unbounded. 
The unboundedness of $\Delta$ implies that $q_{0} \neq 1$. By the Halving lemma (see [23, Theorem 5.2.14]) there exists an infinite sequence $\left(q_{n}\right)$ of mutually orthogonal nonzero projections in $H(N, \beta)$ such that $q_{n} \leq \mathbf{1}-q_{0}$, for every $n \in \mathbb{N}$. Property $(b)$ above implies that $\left.\Delta\right|_{\mathcal{P}\left(H\left(\left(q_{0}+q_{n}\right) M\left(q_{0}+q_{n}\right), \beta\right)\right)}$ is unbounded for all natural $n$.

We claim that $\left.\Delta\right|_{\mathcal{P}\left(H\left(\left(\mathbf{1}-q_{0}\right) M\left(\mathbf{1}-q_{0}\right), \beta\right)\right)}$ is bounded. Indeed, let $\left(r_{n}\right)$ be a sequence of projections in $H(N, \beta)$ such that $\left(r_{n}\right) \searrow 0$ and $r_{n} \leq 1-q_{0}$. The above property $(b)$ of $q_{0}$ also implies that $\left.\Delta\right|_{\mathcal{P}\left(H\left(\left(q_{0}+r_{n}\right) M\left(q_{0}+r_{n}\right), \beta\right)\right)}$ is unbounded for all natural $n$. Since the pair $(H(M, \beta), \Delta)$ satisfies property (3.4) for $p_{0}=1$ in Proposition 3.9, it follows that

$$
\sup \left\{|\Delta(p)|: p \leq \mathbf{1}-q_{0}-r_{n}\right\} \leq 1,(n \in \mathbb{N}) .
$$

The boundedness of the previous set together with the condition $1-q_{0}-r_{n} \nearrow \mathbf{1}-q_{0}$ imply, via Lemma 3.3 that $\left.\Delta\right|_{\mathcal{P}\left(H\left(\left(\mathbf{1}-q_{0}\right) M\left(\mathbf{1}-q_{0}\right), \beta\right)\right)}$ is bounded, which proves the claim.

We have shown that $\left.\Delta\right|_{\mathcal{P}\left(H\left(q_{0} M q_{0}, \beta\right)\right)}$ and $\left.\Delta\right|_{\mathcal{P}\left(H\left(\left(\mathbf{1}-q_{0}\right) M\left(\mathbf{1}-q_{0}\right), \beta\right)\right)}$ are bounded measures. Applying Lemma 3.14 to $\left(q_{0}+q_{n}\right) M\left(q_{0}+q_{n}\right)$ and the projection $p_{0}=q_{0}$, we find a projection $p_{n}$ in $\left(q_{0}+q_{n}\right) M\left(q_{0}+q_{n}\right)$ satisfying $\left|\Delta\left(p_{n}\right)\right|>\frac{n 2^{12 n}}{\delta}$ and $d\left(p_{n}, q_{0}\right) \geq \frac{1}{8}$ (let us observe that since $q_{n} \leq \mathbf{1}-q_{0},\left.\Delta\right|_{\mathcal{P}\left(H\left(q_{n} M q_{n}, \beta\right)\right)}$ is bounded). We define in this way a sequence $\left(p_{n}\right)$ in $\mathcal{P}(H(M, \beta))$.

We shall prove next that, for each natural $n, d\left(p_{n}, \vee_{i \neq n} p_{i}\right) \geq \frac{1}{8}$. To this end, let us pick norm-one elements $\xi \in p_{n}(H)$ and $\eta \in \vee_{i \neq n} p_{i}(H)$ (we regard $M$ as a von Neumann subalgebra of some $B(H)$ ). Having in mind that $p_{n} \leq q_{0}+q_{n}$ with $q_{n} \perp q_{0}(n \in \mathbb{N})$, we deduce that $\vee_{i \neq n} p_{i}(H) \subset q_{0}(H)+\left(\sum_{i \neq n} q_{i}\right)(H)$, and thus, we can write

$$
\eta=\alpha u_{1}+\beta u_{2}
$$

where $\alpha, \beta \geq 0, \alpha^{2}+\beta^{2}=1, u_{1} \in q_{0}(H)$ and $u_{2} \in\left(\sum_{i \neq n} q_{i}\right)(H)$. The images of $q_{0}$ and $\left(\sum_{i \neq n} q_{i}\right)$ are orthogonal in the Hilbert sense, and hence

$$
\begin{gathered}
\|\xi-\eta\|^{2}=\left\|\xi-\alpha u_{1}-\beta u_{2}\right\|^{2}=\left\|\xi-\alpha u_{1}\right\|^{2}+\left\|\beta u_{2}\right\|^{2} \\
\geq\left(\left\|\xi-u_{1}\right\|-\left\|(1-\alpha) u_{1}\right\|\right)^{2}+\beta^{2}=\left(\left\|\xi-u_{1}\right\|-1+\alpha\right)^{2}+1-\alpha^{2} .
\end{gathered}
$$

The last expression in the above inequality defines a function $f(\alpha), \alpha \in[0,1]$, whose extreme values are attained at $\alpha=0$ or $\alpha=1$. Taking $\alpha=0$, we have $\|\xi-\eta\|^{2} \geq\left(\left\|\xi-u_{1}\right\|-1\right)^{2}+$ $1 \geq 1$. In the case $\alpha=1$, we have $\|\xi-\eta\|^{2}=\left\|\xi-u_{1}\right\|^{2} \geq \frac{1}{8^{2}}$, because $u_{1} \in q_{0}(H)$ and $d\left(q_{0}, p_{n}\right) \geq \frac{1}{8}$.

We apply now Lemma 3.4. For each natural $n$, we can find a finite set $\left\{p_{i}^{n}: i=1, \ldots, k_{n}\right\}$ of mutually orthogonal projections in $H(M, \beta)$ satisfying $p_{n}=\sum_{i=1}^{k_{n}} p_{i}^{n}, \varphi\left(p_{i}^{n}\right) \leq \frac{\delta}{2^{11 n}}$, and $k_{n} \leq 2 \frac{2^{11 n}}{\delta}$. The projections in $\left\{p_{i}^{n}: i=1, \ldots, k_{n}\right\}$ are mutually orthogonal, so

$$
\frac{n 2^{12 n}}{\delta}<\left|\Delta\left(p_{n}\right)\right|=\left|\sum_{i=1}^{k_{n}} \Delta\left(p_{i}^{n}\right)\right| \leq \sum_{i=1}^{k_{n}}\left|\Delta\left(p_{i}^{n}\right)\right|,
$$

and therefore there exists $i_{n} \in\left\{1, \ldots, k_{n}\right\}$ such that $\left|\Delta\left(p_{i_{n}}^{n}\right)\right|>n$. So, replacing $p_{n}$ with $p_{i_{n}}^{n}$, it may be assumed that $\varphi\left(p_{n}\right) \leq \frac{\delta}{2^{11 n}}$ and $\left|\Delta\left(p_{n}\right)\right|>n$. 
Now, we take $\varepsilon=\frac{1}{2^{10}}$. Lemma $1(b)$ in [17] asserts that

$$
p_{1} \vee \ldots \vee p_{n} \leq \frac{1}{\varepsilon}\left(p_{1}+p_{2} \vee \ldots \vee p_{n}\right) \leq \frac{1}{\varepsilon} p_{1}+\frac{1}{\varepsilon^{2}}\left(p_{2}+p_{3} \vee \ldots \vee p_{n}\right) \leq \ldots \leq \sum_{k=1}^{n} \frac{1}{\varepsilon^{k}} p_{k}
$$

Therefore,

$$
\varphi\left(p_{1} \vee \ldots \vee p_{n}\right) \leq \sum_{k=1}^{n} \frac{1}{\varepsilon^{k}} \varphi\left(p_{k}\right) \leq \sum_{k=1}^{n} \frac{1}{\varepsilon^{k}} \frac{\delta}{2^{11 k}}=\sum_{k=1}^{n} 2^{10 k} \frac{\delta}{2^{11 k}}<\delta .
$$

This shows that for $r=\bigvee_{n=1}^{\infty} p_{n}, \varphi(r) \leq \delta$ and $\left.\Delta\right|_{\mathcal{P}(H(r M r, \beta))}$ is unbounded, which contradicts that $\Delta$ satisfies property (3.6) for $p_{0}=1$ and $\delta>0$.

\section{2-LOCAL TRIPLE DERIVATIONS ON CONTINUOUS JBW*-TRIPLES}

Recall that a $J B W^{*}$-triple $\mathcal{A}$ is said to be continuous if it has no type I direct summand, and that in this case, up to isometry, $\mathcal{A}$ is a $J W^{*}$-triple with unique decomposition, $\mathcal{A}=$ $H(W, \alpha) \oplus p V$, where $W$ and $V$ are continuous von Neumann algebras, $p$ is a projection in $V, \alpha$ is a *antiautomorphism of $W$ of order 2 , and $H(W, \alpha)=\{x \in W: \alpha(x)=x\}$ (see $[26,(1.20)])$.

We have shown in section 2 that every 2-local triple derivation on $p V$ is a triple derivation. In this section we show that every 2-local triple derivation on $H(W, \alpha)$ is a triple derivation, and hence that every 2-local triple derivation on a continuous $\mathrm{JBW}^{*}$-triple is a triple derivation.

4.1. Triple derivations on $H(M, \beta)$. Assume that $M$ is a continuous von Neumann algebra and $\beta: M \rightarrow M$ is a $\mathbb{C}$-linear ${ }^{*}$-involution (i.e. ${ }^{*}{ }^{*}$-antiautomorphism of order 2 ). In this subsection we shall show that every 2-local triple derivation on the subspace $H(M, \beta)$ of all $\beta$-fixed points in $M$ is a triple derivation.

We begin by taking advantage of the Jordan structure of $H(M, \beta)$ (see the beginning of section 3) to provide a precise description of triple derivations on it.

Let $\delta: H(M, \beta) \rightarrow H(M, \beta)$ be a triple derivation. By [24, Lemma 1 and its proof],

$$
\delta(\mathbf{1})^{*}=-\delta(\mathbf{1}), \text { and } M_{\delta(\mathbf{1})}=\delta\left(\frac{1}{2} \delta(\mathbf{1}), \mathbf{1}\right) \text { is a triple derivation. }
$$

This implies that $D=\delta-M_{\delta(\mathbf{1})}=\delta-\delta\left(\frac{1}{2} \delta(\mathbf{1}), \mathbf{1}\right)$ is a triple derivation satisfying $D(\mathbf{1})=0$. Lemma 2 in [24] implies that $D$ is a Jordan *-derivation on $H(M, \beta)$. Thus, $\left.D\right|_{H(M, \beta)_{s a}}: H(M, \beta)_{s a} \rightarrow H(M, \beta)_{s a}$ is a Jordan derivation on the continuous JBW-algebra $H(M, \beta)_{s a}$. Theorem 3.5 in [42] assures that $\left.D\right|_{H(M, \beta)_{s a}}$ is an inner derivation, that is, there exist $a_{1}, \ldots, a_{m} b_{1}, \ldots, b_{m}$ in $H(M, \beta)_{s a}$ satisfying

$$
D(x)=\sum_{j=1}^{m}\left[M_{a_{j}}, M_{b_{j}}\right](x)=\sum_{j=1}^{m} a_{j} \circ\left(b_{j} \circ x\right)-b_{j} \circ\left(a_{j} \circ x\right)
$$


$=\frac{1}{4} \sum_{j=1}^{m}\left(a_{j} b_{j}-b_{j} a_{j}\right) x-x\left(a_{j} b_{j}-b_{j} a_{j}\right)=\sum_{j=1}^{m}\left[\frac{\left(a_{j} b_{j}-b_{j} a_{j}\right)}{4}, x\right]=\left[\sum_{j=1}^{m} \frac{\left(a_{j} b_{j}-b_{j} a_{j}\right)}{4}, x\right]$,

for every $x \in H(M, \beta)_{s a}$. If we denote $a=\sum_{j=1}^{m} \frac{\left(a_{j} b_{j}-b_{j} a_{j}\right)}{4} \in M$, then $\beta(a)=-a$ and $a^{*}=-a$ (just observe that $\beta\left(a_{j}\right)=a_{j}, a_{j}^{*}=a_{j}, \beta\left(b_{j}\right)=b_{j}$, and $b_{j}^{*}=b_{j}$, for every $j$ ), and, by (4.2),

$$
\delta(x)=[a, x]+\delta(1) \circ x,
$$

for every $x \in H(M, \beta)_{s a}$. The following proposition summarizes the above facts.

Proposition 4.1. Let $M$ be a continuous von Neumann algebra and let $\beta: M \rightarrow M$ be $a \mathbb{C}$-linear ${ }^{*}$-involution. Then for every triple derivation $\delta$ on the $J B W^{*}$-algebra $H(M, \beta)$, of all $\beta$-fixed points in $M$, there exist $a, b \in M$ with $a^{*}=-a, b^{*}=-b, \beta(a)=-a$ and $\beta(b)=b=\delta(\mathbf{1})$, satisfying

$$
\delta(x)=[a, x]+b \circ x,
$$

for every $x \in H(M, \beta)$. Consequently, every triple derivation on $H(M, \beta)$ admits an extension to a triple derivation on $M$.

4.2. 2-local triple derivations on $H(M, \beta)$. Let $J$ be a $\mathrm{JBW}^{*}$-subalgebra of a von Neumann algebra $M$. Suppose that $J$ contains the unit of $M$. Given a self-adjoint element $z \in J$, the $\mathrm{JBW}^{*}$-subalgebra, $\mathcal{W}^{*}(z)$, of $J$ generated by $z$ and the unit element is an associative $\mathrm{JBW}^{*}$-algebra isometrically isomorphic to a commutative von Neumann algebra (cf. [23, Lemma 4.1.11]). It is known that $\mathcal{W}^{*}(z)$ coincides with the abelian von Neumann subalgebra of $M$ generated by the element $z$ and the unit element.

Let $\Delta: H(M, \beta) \rightarrow H(M, \beta)$ be a (not necessarily linear nor continuous) 2-local triple derivation. By (4.1) we deduce that $\Delta(\mathbf{1})^{*}=-\Delta(\mathbf{1})$ and $M_{\Delta(\mathbf{1})}=\delta\left(\frac{1}{2} \Delta(\mathbf{1}), \mathbf{1}\right)$ is a triple derivation. Replacing $\Delta$ with $\Delta-\delta\left(\frac{1}{2} \Delta(\mathbf{1}), \mathbf{1}\right)$ we can assume that our 2-local triple derivation satisfies $\Delta(\mathbf{1})=0$. Having in mind the description provided by the above Proposition 4.1, the arguments given in [32, Lemmas 2.2, 2.3, and 2.6] can be literally adapted to prove the following:

Lemma 4.2. Let $M$ be a continuous von Neumann algebra and let $\beta: M \rightarrow M$ be a $\mathbb{C}$ linear ${ }^{*}$-involution. Suppose that $\Delta: H(M, \beta) \rightarrow H(M, \beta)$ is a (not necessarily linear nor continuous) 2-local triple derivation. Then the following statements hold:

(a) If $\Delta(\mathbf{1})=0$, then $\Delta(x)=\Delta(x)^{*}$ for all $x \in H(M, \beta)_{\text {sa }}$;

(b) If $\Delta(\mathbf{1})=0$, then for every $x, y \in H(M, \beta)_{\text {sa }}$ there exists a skew-hermitian element $a_{x, y} \in M$ with $\beta\left(a_{x, y}\right)=-a_{x, y}$ such that $\Delta(x)=\left[a_{x, y}, x\right]$, and $\Delta(y)=\left[a_{x, y}, y\right]$;

(c) Let $z \in H(M, \beta)$ be a self-adjoint element and let $\mathcal{W}^{*}(z)=\{z\}^{\prime \prime}$ be the abelian von Neumann subalgebra of $M$ generated by the element $z$ and the unit element. Then there exist skew-hermitian elements $a_{z}, b_{z} \in M$, depending on $z$, such that

$$
\Delta(x)=\left[a_{z}, x\right]+b_{z} \circ x=a_{z} x-x a_{z}+\frac{1}{2}\left(b_{z} x+x b_{z}\right)
$$

for all $x \in \mathcal{W}^{*}(z) \subseteq H(M, \beta)$. In particular, $\Delta$ is linear and continuous on $\mathcal{W}^{*}(z)$. 
The results in Lemma 4.2 will be now applied to obtain a Jordan version of [32, Proposition 2.7]. Given a $\mathrm{JBW}^{*}$-algebra $J$ whose lattice of projections is denoted by $\mathcal{P}(J)$, and a Banach space $X$, a finitely additive $X$-valued measure on $\mathcal{P}(J)$ is defined in the same way as in the case of a von Neumann algebra, namely, a mapping $\mu: \mathcal{P}(J) \rightarrow X$ satisfying

$$
\mu\left(\sum_{i=1}^{n} p_{i}\right)=\sum_{i=1}^{n} \mu\left(p_{i}\right)
$$

for every family $p_{1}, \ldots, p_{n}$ of mutually orthogonal projections in $J$.

Let $\left(p_{i}\right)_{i \in I}$ be a family of mutually orthogonal projections in a $\mathrm{JBW}^{*}$-algebra $J$. The series $\sum_{i \in I} p_{i}$ is summable with respect to the strong* topology of $J$, and we further know that the limit $p=$ strong $^{*}-\sum_{i \in I} p_{i}$ is another projection in $J$ (cf. [23, remark 4.2.9]). In particular, $\sum_{i \in I} p_{i}$ is summable with respect to the weak* topology of $J$ and strong*- $\sum_{i \in I} p_{i}=$ weak $^{*}-\sum_{i \in I} p_{i}$.

Let $J_{1}$ and $J_{2}$ be $\mathrm{JBW}^{*}$-algebras, and let $\tau$ denote the norm, the weak* or the strong* topology of $J_{1}$. As in the case of von Neumann algebras, a mapping $\mu: J_{1} \rightarrow J_{2}$ is said to be $\tau$-completely additive (respectively, countably or sequentially $\tau$-additive) when

$$
\mu\left(\sum_{i \in I} p_{i}\right)=\tau-\sum_{i \in I} \mu\left(p_{i}\right)
$$

for every family (respectively, sequence) $\left\{p_{i}\right\}_{i \in I}$ of mutually orthogonal projections in $J_{1}$.

We can easily obtain now a Jordan version of [32, Proposition 2.7].

Proposition 4.3. Let $M$ be a continuous von Neumann algebra and let $\beta: M \rightarrow M$ be $a \mathbb{C}$-linear ${ }^{*}$-involution. Let $\Delta: H(M, \beta) \rightarrow H(M, \beta)$ be a (not necessarily linear nor continuous) 2-local triple derivation. Then the following statements hold:

(a) The restriction $\left.\Delta\right|_{\mathcal{P}(J)}$ is sequentially strong*-additive, and consequently sequentially weak ${ }^{*}$-additive;

(b) $\left.\Delta\right|_{\mathcal{P}(J)}$ is weak ${ }^{*}$-completely additive, i.e.,

$$
\Delta\left(\text { weak }^{*}-\sum_{i \in I} p_{i}\right)=\text { weak }^{*}-\sum_{i \in I} \Delta\left(p_{i}\right)
$$

for every family $\left(p_{i}\right)_{i \in I}$ of mutually orthogonal projections in $J$.

Proof. $(a)$ Let $\left(p_{n}\right)_{n \in \mathbb{N}}$ be a sequence of mutually orthogonal projections in $H(M, \beta)$. Let us consider the element $z=\sum_{n \in \mathbb{N}} \frac{1}{n} p_{n}$. Let $\mathcal{W}^{*}(z)$ be the $\mathrm{JBW}^{*}$-subalgebra of $H(M, \beta)$ 
generated by $z$. By Lemma 4.2(c), there exist skew-hermitian elements $a_{z}, b_{z} \in M$ with $\beta\left(a_{z}\right)=-a_{z}$ and $\beta\left(b_{z}\right)=b_{z}$, satisfying

$$
T(x)=\left[a_{z}, x\right]+b_{z} \circ x,
$$

for all $x \in \mathcal{W}^{*}(z)$.

The elements $\sum_{n=1}^{\infty} p_{n}$, and $p_{m}$ belong to $\mathcal{W}^{*}(z)$, for all $m \in \mathbb{N}$. The reader should be warned that $a_{z}$ might not belong to $H(M, \beta)$. In any case, the product of $M$ is jointly strong* continuous on bounded sets, and by [8, Corollary] $\left.S^{*}\left(M, M_{*}\right)\right|_{H(M, \beta)} \equiv S^{*}\left(H(M, \beta), H(M, \beta)_{*}\right)$. Therefore,

$$
\begin{gathered}
\Delta\left(S^{*}\left(M, M_{*}\right)-\sum_{n=1}^{\infty} p_{n}\right)=\left[a_{z}, S^{*}\left(M, M_{*}\right)-\sum_{n=1}^{\infty} p_{n}\right]+b_{z} \circ S^{*}\left(M, M_{*}\right)-\left(\sum_{n=1}^{\infty} p_{n}\right) \\
=S^{*}\left(M, M_{*}\right)-\sum_{n=1}^{\infty}\left[a_{z}, p_{n}\right]+S^{*}\left(M, M_{*}\right)-\sum_{n=1}^{\infty} b_{z} \circ p_{n}=S^{*}\left(M, M_{*}\right)-\sum_{n=1}^{\infty} \Delta\left(p_{n}\right),
\end{gathered}
$$

i.e. $\left.\Delta\right|_{\mathcal{P}(M)}$ is a countably or sequentially strong* additive mapping.

(b) As we have commented above, the strong*-topology of the $\mathrm{JBW}^{*}$-algebra $H(M, \beta)$ coincides with the restriction to $H(M, \beta)$ of the strong*-topology of $M$. When in the proof of [32, Proposition 2.7](b), we replace [32, Lemmas 2.2 and 2.3] with Lemma 4.2 (and having in mind the conclusion of Proposition 4.1), the arguments remaind valid to obtain the desired statement here.

Let $\Delta: H(M, \beta) \rightarrow H(M, \beta)$ be a (not necessarily linear nor continuous) 2-local triple derivation, where $M$ is a continuous von Neumann algebra and $\beta: M \rightarrow M$ is a $\mathbb{C}$-linear ${ }^{*}$-involution. For each normal state $\phi \in H(M, \beta)_{*}$ ( or $\phi \in M_{*}$ ), Proposition 4.3 implies that the mapping $\left.\phi \circ \Delta\right|_{\mathcal{P}(H(M, \beta))}: \mathcal{P}(H(M, \beta)) \rightarrow \mathbb{C}$ is a completely additive measure. We conclude from Theorem 3.1, and from the arbitrariness of $\phi$ together with the uniform boundedness principle, that $\left.\Delta\right|_{\mathcal{P}(H(M, \beta))}: \mathcal{P}(H(M, \beta)) \rightarrow \mathbb{C}$ is a bounded weak*-completely additive measure. An appropriate Jordan version of the Bunce-Wright-Mackey-Gleason theorem (see Theorem 3.2) implies the existence of a bounded linear operator $G: H(M, \beta) \rightarrow H(M, \beta)$ satisfying that $G(p)=\Delta(p)$ for every $p \in \mathcal{P}(H(M, \beta))$.

Let us pick a self-adjoint element $z$ in $H(M, \beta)$. By Lemma 4.2(c), there exist skewhermitian elements $a_{z}, b_{z} \in M$, with $\beta\left(a_{z}\right)=-a_{z}$ and $\beta\left(b_{z}\right)=b_{z}$, such that $\Delta(x)=$ $\left[a_{z}, x\right]+b_{z} \circ x$, for every $x \in \mathcal{W}^{*}(z)$, the $\mathrm{JBW}^{*}$-subalgebra of $H(M, \beta)$ generated by $z$. Since $\left.G\right|_{\mathcal{W}^{*}(z)}$ and $\left.\Delta\right|_{\mathcal{W}^{*}(z)}$ are bounded linear operators from $\mathcal{W}^{*}(z)$ to $M$, which coincide on the set of projections of $\mathcal{W}^{*}(z)$, and every self-adjoint element in $\mathcal{W}^{*}(z)$ can be approximated in norm by finite linear combinations of mutually orthogonal projections in $\mathcal{W}^{*}(z)$, we conclude that $\Delta(x)=G(x)$ for every $x \in \mathcal{W}^{*}(z)$, and hence

$$
\Delta(z)=G(z), \text { for every } z \in H(M, \beta)_{s a},
$$

in particular, $\Delta$ is additive on $H(M, \beta)_{s a}$. This proves the following Proposition. 
Proposition 4.4. Let $\Delta: H(M, \beta) \rightarrow H(M, \beta)$ be a (not necessarily linear nor continuous) 2-local triple derivation, where $M$ is a continuous von Neumann algebra and $\beta: M \rightarrow M$ is a $\mathbb{C}$-linear ${ }^{*}$-involution. Then the restriction $\left.\Delta\right|_{H(M, \beta)_{s a}}$ is additive.

Lemma 4.5. Let $\Delta: H(M, \beta) \rightarrow H(M, \beta)$ be a (not necessarily linear nor continuous) 2-local triple derivation, where $M$ is a continuous von Neumann algebra and $\beta: M \rightarrow M$ is a $\mathbb{C}$-linear ${ }^{*}$-involution. Suppose $\Delta(\mathbf{1})=0$. Then there exists a skew-hermitian element $a \in M$ such that $\beta(a)=-a$, and $\Delta(x)=[a, x]$, for all $x \in H(M, \beta)_{\text {sa }}$.

Proof. Let $x \in M_{s a}$. By Lemma 4.2(c) there exist a skew-hermitian element $a_{x, x^{2}} \in M$ such that $\beta\left(a_{x, x^{2}}\right)=-a_{x, x^{2}}$, and $\Delta(x)=\left[a_{x, x^{2}}, x\right], \Delta\left(x^{2}\right)=\left[a_{x, x^{2}}, x^{2}\right]$.

Thus,

$$
\Delta\left(x^{2}\right)=\left[a_{x, x^{2}}, x^{2}\right]=\left[a_{x, x^{2}}, x\right] x+x\left[a_{x, x^{2}}, x\right]=2 \Delta(x) \circ x .
$$

By Proposition 4.4 and Lemma 4.2(a), $\left.\Delta\right|_{H(M, \beta)_{s a}}: H(M, \beta)_{s a} \rightarrow H(M, \beta)_{s a}$ is a real linear mapping. Now, we consider the linear extension $\hat{\Delta}$ of $\left.\Delta\right|_{H(M, \beta)_{s a}}$ to $H(M, \beta)$ defined by

$$
\hat{\Delta}\left(x_{1}+i x_{2}\right)=T\left(x_{1}\right)+i T\left(x_{2}\right), x_{1}, x_{2} \in H(M, \beta)_{s a} .
$$

Taking into account the homogeneity of $\Delta$, Proposition 4.4 and the identity (4.5), we deduce that $\hat{\Delta}$ is a Jordan *-derivation (and hence, a triple derivation) on $H(M, \beta)$. Proposition 4.1 implies the existence of a skew-symmetric element $a \in M$ such that $\beta(a)=-a$ and $\hat{\Delta}(x)=[a, x]$ for all $x \in H(M, \beta)$. In particular, $\Delta(x)=[a, x]$ for all $x \in H(M, \beta)_{s a}$, which completes the proof.

We now prove the main result of this section.

Theorem 4.6. Let $\Delta: H(M, \beta) \rightarrow H(M, \beta)$ be a (not necessarily linear nor continuous) 2-local triple derivation, where $M$ is a continuous von Neumann algebra and $\beta: M \rightarrow M$ is a $\mathbb{C}$-linear ${ }^{*}$-involution. Then $\Delta$ is a linear and continuous triple derivation.

Proof. From (4.1) we know that $\Delta(\mathbf{1})^{*}=-\Delta(\mathbf{1})$, and $M_{\Delta(\mathbf{1})}=\delta\left(\frac{1}{2} \Delta(\mathbf{1}), \mathbf{1}\right)$ is a triple derivation. Replacing $\Delta$ with $\Delta-\delta\left(\frac{1}{2} \Delta(\mathbf{1}), \mathbf{1}\right)$ we can assume that $\Delta(\mathbf{1})=0$. By Lemma 4.5 there exists a skew-hermitian element $a \in M$ such that $\beta(a)=-a$, and $\Delta(x)=[a, x]$, for all $x \in H(M, \beta)_{s a}$. Observe that the mapping $\widehat{\Delta}=\Delta-[a,$.$] is a 2-local triple derivation$ on $H(M, \beta)_{s a}$ satisfying $\left.\widehat{\Delta}\right|_{H(M, \beta)_{s a}} \equiv 0$.

We shall finally prove that $\widehat{\Delta}=0$. This result follows from a direct adaptation of the arguments in [32, Lemma 2.16], we include here a sketch of the proof for completeness reasons.

Let $x \in H(M, \beta)$ be an arbitrary element and let $x=x_{1}+i x_{2}$, where $x_{1}, x_{2} \in H(M, \beta)_{s a}$. Since $\widehat{\Delta}$ is homogeneous, by passing to the element $\left(1+\left\|x_{2}\right\|\right)^{-1} x$ if necessary, we can suppose that $\left\|x_{2}\right\|<1$. In this case the element $y=1+x_{2}$ is positive and invertible. Take skew-hermitian elements $a_{x, y}, b_{x, y} \in M$ such that $\beta\left(a_{x, y}\right)=-a_{x, y}, \beta\left(b_{x, y}\right)=b_{x, y}$, and

$$
\widehat{\Delta}(x)=\left[a_{x, y}, x\right]+b_{x, y} \circ x \text {, and } \widehat{\Delta}(y)=\left[a_{x, y}, y\right]+b_{x, y} \circ y .
$$


Since $\widehat{\Delta}(y)=0$, we get $\left[a_{x, y}, y\right]+b_{x, y} \circ y=0$. Lemma 2.4 in [32] implies that $\left[a_{x, y}, y\right]=0$ and $i b_{x, y} \circ y=0$. Having in mind that $y$ is positive and invertible, and that $i b_{x, y}$ is hermitian, $\left[32\right.$, Lemma 2.5] proves that $b_{x, y}=0$.

The condition $0=\left[a_{x, y}, y\right]=\left[a_{x, y}, \mathbf{1}+x_{2}\right]=\left[a_{x, y}, x_{2}\right]$, implies

$$
\widehat{\Delta}(x)=\left[a_{x, y}, x\right]+b_{x, y} \circ x=\left[a_{x, y}, x_{1}+i x_{2}\right]=\left[a_{x, y}, x_{1}\right]
$$

which shows that

$$
\widehat{\Delta}(x)^{*}=\left[a_{x, y}, x_{1}\right]^{*}=\left[x_{1}, a_{x, y}^{*}\right]=\left[x_{1},-a_{x, y}\right]=\left[a_{x, y}, x_{1}\right]=\widehat{\Delta}(x) .
$$

The arbitrariness of $x \in H(M, \beta)$ implies that $\widehat{\Delta}(x)=0$, as desired.

Since every element in a closed ideal of a $\mathrm{JB}^{*}$-triple can be written as a cube of an element in that ideal, it is clear that a triple derivation leaves closed ideals invariant. Hence the same is true for 2-local triple derivations. Thus, by invoking the structure theorem of continuous $\mathrm{JBW}^{*}$-triples stated at the beginning of this section, and combining Theorems 2.3 and 4.6, we obtain the second main result of this paper.

Theorem 4.7. Let $\Delta: A \rightarrow A$ be a (not necessarily linear nor continuous) 2-local triple derivation, where $A$ is a continuous $J B W^{*}$-triple. Then $\Delta$ is a linear and continuous triple derivation.

Problem 4.8. Does Theorem 3.1 remain valid when $H(M, \beta)$ is replaced by an arbitrary $J B W^{*}$-algebra without summands of type $I_{n}$ ?

Problem 4.9. Is Theorem 4.7 valid for

(a): $J B W^{*}$-triples of type I? (See Corollary 2.12)

(b): reversible $J B W^{*}$-algebras?

(c): 2-local triple derivations with values in a Jordan triple module?

(d): 2-local triple derivations on various algebras of measurable operators?

(e): real $J B W^{*}$-triples?

(f): complex and real JB*-triples?

\section{REFERENCES}

[1] S. Ayupov, A new proof of the existence of traces on Jordan operator algebras and real von Neumann algebras, J. Funct. Anal. 84, no. 2, 312-321 (1989).

[2] S. Ayupov, K. Kudaybergenov, Derivations, local, and 2-local derivations on algebras of measurable operators, In: Proceedings of the USA-Uzbekistan conference on Analysis and Mathematical Physics, May 20-23, 2014, Fullerton, California, Contemporary Mathematics, to appear.

[3] S. Ayupov, K. Kudaybergenov, 2-local derivations on von Neumann algebras. Positivity 19, no. 3, 445455 (2015).

[4] S. Ayupov, K. Kudaybergenov, A. M. Peralta, A survey on local and 2-local derivations on C*-algebras and von Neumann algebras, In: Proceedings of the USA-Uzbekistan conference on Analysis and Mathematical Physics, May 20-23, 2014, Fullerton, California, Contemporary Mathematics, to appear.

[5] S. Ayupov, A. Rakhimov, S. Usmanov: Jordan, Real and Lie Structures in Operator Algebras. Kluwer, 1997. 
[6] B. Blackadar, Operator algebras. Theory of $C^{*}$-algebras and von Neumann algebras. Encyclopaedia of Mathematical Sciences, 122. Operator Algebras and Non-commutative Geometry, III. Springer-Verlag, Berlin, 2006.

[7] M. Bohata, J, Hamhalter, O. F. K. Kalenda, Decompositions of preduals of JBW and JBW*-algebras, arXiv:1511.01086 (2015)

[8] L. J. Bunce, Norm preserving extensions in JBW*-triple preduals, Quart. J. Math. 52 (2), 133-136 (2001).

[9] L. J. Bunce, J. D. M. Wright, Continuity and linear extensions of quantum measures on Jordan operator algebras, Math. Scand. 64, 300-306 (1989).

[10] L. J. Bunce, J. D. M. Wright, The Mackey-Gleason problem, Bull. Amer. Math. Soc. 26, 288-293 (1992).

[11] L. J. Bunce, J. D. M. Wright, The Mackey-Gleason problem for vector measures on projections in von Neumann algebras, J. London Math. Soc. 49, 133-149 (1994).

[12] M. Burgos, F. J. Fernández-Polo, A. M. Peralta, Local triple derivations on C*-algebras and JB*-triples. Bull. Lond. Math. Soc. 46, no. 4, 709-724 (2014).

[13] M. Burgos, F.J. Fernández-Polo, J.J. Garcés and A.M. Peralta, A Kowalski-Slodkowski theorem for 2local *-homomorphisms on von Neumann algebras, Rev. R. Acad. Cienc. Exactas Fís. Nat. Ser. A Math. RACSAM 109, no. 2, 551-568 (2015).

[14] M. Burgos, F.J. Fernández-Polo, J.J. Garcés, A.M. Peralta, 2-local triple homomorphisms on von Neumann algebras and JBW*-triples, J. Math. Anal. Appl. 426, 43-63 (2015).

[15] C.-H. Chu, Jordan structures in geometry and analysis. Cambridge Tracts in Mathematics, 190. Cambridge University Press, Cambridge, 2012. x+261 pp

[16] T. Cooney, M. Junge, M. Navascués, D. Pérez-García, I. Villanueva, Joint system quantum descriptions arising from local quantumness, Comm. Math. Phys. 322, no. 2, 501-513 (2013).

[17] S. Dorofeev, On the problem of boundedness of a signed measure on projections of a von Neumann algebra, J. Funct. Anal. 103, 209-216 (1992).

[18] A. Dvurecenskij, Gleason's theorem and its applications. Mathematics and its Applications (East European Series), 60. Kluwer Academic Publishers Group, Dordrecht; Ister Science Press, Bratislava, 1993. ISBN: 0-7923-1990-7

[19] C. M. Edwards, G. T. Rüttimann, Exposed faces of the unit ball in a JBW*-triple, Math. Scand. 82, 287-304 (1998).

[20] C. M. Edwards, G. T. Rüttimann, Gleason's theorem for rectangular JBW*-triples, Comm. Math. Phys. 203, no. 2, 269-295 (1999).

[21] A. M. Gleason, Measures on the closed subspaces of a Hilbert space, J. Math. Mech. 6, 885-893 (1957) .

[22] J. Hamhalter, Quantum Measure Theory, Kluwer, 2003.

[23] H. Hanche-Olsen, E. Størmer, Jordan operator algebras, Monographs and Studies in Mathematics 21, Pitman, London-Boston-Melbourne 1984.

[24] T. Ho, J. Martínez-Moreno, A. M. Peralta, B. Russo, Derivations on real and complex JB*-triples, J. London Math. Soc. (2) 65, no. 1, 85-102 (2002).

[25] G. Horn, Classification of JBW*-triples of type I. Math. Z. 196 (1987), no. 2, 271-291.

[26] G. Horn, E. Neher, Classification of continuous JBW*-triples, Trans. Amer. Math. Soc. 306, 553-578 (1988).

[27] B. E. Johnson, Local derivations on $\mathrm{C}^{*}$-algebras are derivations, Trans. Amer. Math. Soc. 353, 313-325 (2001).

[28] R. V. Kadison, Local derivations, J. Algebra 130, 494-509 (1990).

[29] R. V. Kadison, J. Ringrose, Fundamentals of the theory of operator algebras, Volume II, Birkhauser, Boston, 1986.

[30] R. V. Kadison, J. Ringrose, Fundamentals of the theory of operator algebras, Volume IV, Birkhauser, Boston, 1992.

[31] W. Kaup, A Riemann mapping theorem for bounded symmetric domains in complex Banach spaces. Math. Z. 183, no. 4, 503-529 (1983). 
[32] K. Kudaybergenov, T. Oikhberg, A. M. Peralta, B. Russo, 2-Local triple derivations on von Neumann algebras, Illinois J. Math. 58, no. 4, 1055-1069 (2014).

[33] D. R. Larson, A. R. Sourour, Local derivations and local automorphisms of B(X), Proc. Sympos. Pure Math. 51, Part 2, Providence, Rhode Island 1990, pp. 187-194.

[34] M. Mackey, Local derivations on Jordan triples, Bull. London Math. Soc. 45, no. 4, 811-824 (2013). doi: 10.1112/blms/bdt007

[35] M. S. Matveichuk, Linearity of a charge on a lattice of orthoprojectors. (Russian) Izv. Vyssh. Uchebn. Zaved. Mat. 1995, no. 9, 48-66; translation in Russian Math. (Iz. VUZ) 39, no. 9, 46-64 (1995).

[36] L. Molnár, Local automorphisms of some quantum mechanical structures, Lett. Math. Phys. 58, no. 2, 91-100 (2001).

[37] O. Rudolph, J.D.M. Wright, On unentangled Gleason theorems for quantum information theory, Lett. Math. Phys. 52, no. 3, 239-245 (2000).

[38] P. Šemrl, Local automorphisms and derivations on B(H), Proc. Amer. Math. Soc. 125, 2677-2680 (1997).

[39] A. N. Sherstnev, Methods of bilinear forms in noncommutative theory of measure and integral, Moscow, Fizmatlit, 2008, 256 pp.

[40] Takesaki, M., Theory of operator algebras I, Springer Verlag, New York, 1979.

[41] D. M. Topping, Jordan algebras of self-adjoint operators, Mem. Amer. Math. Soc., 53, 1965. 48pp

[42] H. Upmeier, Derivations of Jordan C*-algebras, Math. Scand. 46, 251-264 (1980).

[43] J.D.M. Wright, Decoherence functionals for von Neumann quantum histories: boundedness and countable additivity, Comm. Math. Phys. 191, no. 3, 493-500 (1998).

E-mail address: hamhalte@math.feld.cvut.cz

Czech Technical University, Faculty of Electrical Engineering, Technicka 2, 166 27, Prague 6, CZech Republic

E-mail address: karim2006@mail.ru

Ch. Abdirov 1, Department of Mathematics, Karakalpak State University, Nukus 230113 , UZBEKISTAN

E-mail address: aperalta@ugr.es

Departamento de Análisis Matemático, Facultad de Ciencias, Universidad de Granada, 18071 GRANADA, SPAin.

E-mail address: brusso@uci.edu

Department of Mathematics, UC IRVINe, IRVine CA, USA 